\title{
亲核氟源参与的芳环氟-18 标记反应研究进展
}

\author{
朱源陈乐园侯文涁*李神亮* \\ (中国医学科学院北京协和医学院放射医学研究所 天津市放射医学与分子核医学重点实验室 天津 300192)
}

\begin{abstract}
摘要 氟 18 是用于临床和临床前研究的正电子发射计算机断层扫描(PET)放射性药物中最常用的放射性同位素, 至今 已发现发展出多种标记方法. 亲核性 ${ }^{18} \mathrm{~F}$ 氟源由于易于操作且具有高放射性比活度, 在氟标记反应中得到广泛应用. 同 时, 引入氟原子可调节药物分子的空间构象和电性, 也能影响其极性、亲脂性和解离常数等药物代谢动力学参数. 芳香 环本身代谢稳定性好, 是理想的氟标记载体. 亲核型氟源参与的芳香环氟-18 标记反应为放射性药物的标记, 具有高 效、操作简便、反应条件温和及原子经济性等优点, 为氟-18 标记提供了一条新途径. 根据标记前体结构的不同, 综述 了近年来亲核型氟源参与的芳香环氟-18标记方法领域研究进展，同时探讨了部分反应的机理，并对本领域未来发展进 行了展望.
\end{abstract}

关键词＼cjkstart氟-18；亲核取代反应；标记反应；放射性药物；正电子发射计算机断层扫描

\section{Recent Progress in Nucleophilic Fluoride Mediated Fluorine-18 Labeling of Arenes and Heteroarenes}

\author{
Zhu, Yuan Chen, Leyuan Hou, Wenbin* Li, Yiliang* \\ (Tianjin Key Laboratory of Radiation Medicine and Molecular Nuclear Medicine, Institute of Radiation Medicine, \\ Peking Union Medical College \& Chinese Academy of Medical Sciences, Tianjin 300192)
}

\begin{abstract}
Fluorine-18 is the most frequently used radioisotope in positron emission tomography radiopharmaceuticals for both clinical and preclinical researches. A variety of labeling methodologies have also been developed in recent years. For most purposes, nucleophilic ${ }^{18} \mathrm{~F}$-fluoride is preferentially used for ${ }^{18} \mathrm{~F}$-labeling because this reagent is easy to handle and made available with high specific activity. Meanwhile, fluorine substitution has also served the purpose of modulating conformational and stereoelectronic properties, and favorably influences pharmacokinetic parameters such as polarity, lipophilicity and $\mathrm{p} K_{\mathrm{a}}$ values. Arenes and heteroarenes are privileged candidates for ${ }^{18} \mathrm{~F}$-incorporation as they are metabolically robust and therefore widely used for ${ }^{18} \mathrm{~F}$-labeling. Nucleophilic fluoride mediated fluorine-18 labeling reaction has emerged as a promising green and efficient synthetic tool and provides a novel approach for ${ }^{18} \mathrm{~F}$-labeling. The recent developments in nucleophilic fluoride mediated fluorine-18 labeling of arenes and heteroarenes are summarized on the basis of different labeling precursor, including phenols, aryl iodoniums, aryl sulfoniums, aromatic metallic compounds and $\mathrm{C}\left(\mathrm{sp}^{2}\right)-\mathrm{H}$ bond. The scope of labeling substrate, some application for radiopharmaceuticals and mechanism of several reactions are also discussed.

Keywords fluorine-18; nucleophilic substitution reaction; labeling reaction; radiopharmaceuticals; positron emission tomography
\end{abstract}

正电子发射计算机断层扫描(positron emission tomography, PET) 是一种非侵入性分子成像技术, 可以用 于探测体内的生物化学过程, 监测疾病的进展, 评估药 物疗效等方面 ${ }^{[1-5]}$. PET 的发展着重于高效简便地合成靶 向放射性示踪剂. 同时要使放射性药物向临床成功转
化, 关键不但在于 PET 示踪剂本身具有的药理学特性, 更重要的是实现一种高效实用、能广泛应用的放射性标 记方法. 同时，选择适宜的核素应用于药物的标记也至 关重要.

氟-18 (Fluorine-18, ${ }^{18} \mathrm{~F}$ ) 是应用最广的 PET 核素, 具

* Corresponding authors. E-mail: houwenbin@irm-cams.ac.cn; liyiliang@irm-cams.ac.cn

Received October 21, 2020; revised December 29, 2020; published online January 14, 2021.

Project supported by the Chinese Academy of Medical Sciences Innovation Fund for Medical Sciences (Nos. 2016-I2M-3-022, 2017-I2M-3-019), the Fundamental Research Funds for the Central Universities (No. 3332018117), and the Science and Technology Project of Tianjin of China (No. 18ZXXYSY00110). 中国医学科学院医学与健康科技创新工程(Nos. 2016-I2M-3-022, 2017-I2M-3-019)、中央高校基本科研业务费专项资金(No. 3332018117)、天津市科技 计划(No. 18ZXXYSY00110)资助项目. 
有相对长的半衰期(109.8 min), 这一特点可用于多步合 成, 扩宽标记反应的适用范围, 同时也可在没有回旋加 速器的场景使用. 在芳环上引入氟原子是常用的研究药 物代谢的策略, 引入氟会减慢药物代谢酶对药物分子的 代谢降解, 并且可以定量地检测代谢副产物. 临床上大 量使用 2-脱氧 $-2-\left[{ }^{18} \mathrm{~F}\right]$ 氟- $D$ - 葡萄糖 $\left(\left[{ }^{18} \mathrm{~F}\right] \mathrm{FDG}\right)$ 等放射性 药物诊断癌症, 心血管疾病和神经系统疾病. 氟-18 通 常来自回旋加速器产生的 $\left[{ }^{18} \mathrm{~F}\right] \mathrm{F}_{2}$ 和 $\left[{ }^{18} \mathrm{~F}\right] \mathrm{F}^{-}$. 在标记反应 中, 两者分别作为亲电试剂和亲核试剂与底物发生反 应, 故分别成为亲电型氟源和亲核型氟源. 使用亲电型 氟源如 $\left[{ }^{18} \mathrm{~F}\right] \mathrm{F}_{2}$ 作为标记的氟-18 来源时, ${ }^{18} \mathrm{~F} /{ }^{19} \mathrm{~F}$ 比率(放 射性比活度)大大低于使用亲核型 $\left[{ }^{18} \mathrm{~F}\right]$ 氟化物时的比活 度, 而高放射性比活度的放射性药物才能得到有临床意 义的 PET 图像. 这些优异的特点促进了新型高效亲核型 芳环放射性氟标记方法的发展.

本文综述了近年来有关 ${ }^{18} \mathrm{~F}$ 放射性标记的方法, 着 重介绍亲核型氟源参与的芳环上单氟原子标记反应, 按 照氟标记反应的反应类型及标记前体的化学结构进行 分类, 总结了不同结构底物氟标记方法的差异, 并讨论 了一系列有代表性和临床相关的小分子 PET 药物的合 成方法, 希望能对本领域的研究起到一定的辅助和促进 作用.
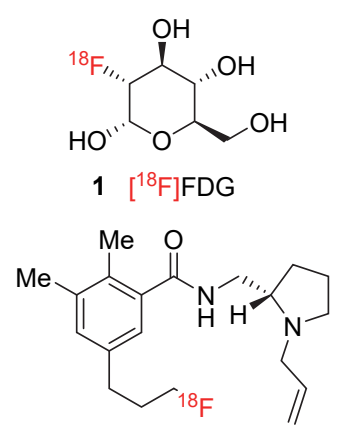

$3 \quad\left[{ }^{18} \mathrm{~F}\right]$ Fallypride<smiles>N[C@@H](Cc1cc(O)c(O)cc1Br)C(=O)O</smiles><smiles>Cc1nc2n(c(=O)c1CCN1CCC(C(=O)c3ccc(F)cc3)CC1)CCS2</smiles>

$7 \quad\left[{ }^{18} \mathrm{~F}\right]$ Setoperone

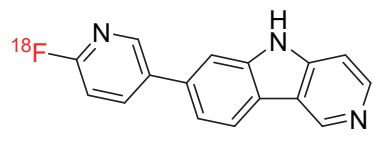

$6\left[{ }^{18} \mathrm{~F}\right] \mathrm{AV}-1451$

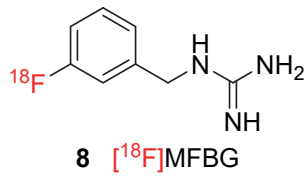

图 1 一些重要的放射性药物

Figure 1 Representative examples of radiopharmaceuticals

\section{1 芳香亲核氟-18 标记反应}

亲核取代 $\left(\mathrm{S}_{\mathrm{N}} \mathrm{Ar}\right)$ 广泛应用于芳香族分子的官能化 ${ }^{[6]}$, 使用放射性氟标记试剂进行的芳香亲核取代 $\left(\mathrm{S}_{\mathrm{N}} \mathrm{Ar}\right)$ 是一 种常见的形成 $\mathrm{C}\left(\mathrm{sp}^{2}\right)-{ }^{18} \mathrm{~F}$ 键的方法(Eq. 1). 许多亲核试 剂表现出 $\mathrm{S}_{\mathrm{N}} \mathrm{Ar}$ 反应活性, 并且反应操作简单、可靠, 可 以应用大规模合成过程. 优秀的氟-18 标记前体一般需 要有好的离去基团和特定的芳香环电性. 在 $\mathrm{S}_{\mathrm{N}} \mathrm{Ar}$ 过程 中, 亲核试剂对带有离去基的碳原子进攻, 会产生一种 带负电荷的中间产物, 称为 Meisenheimer 复合物, 邻位 或对位存在活性基团(通常是吸电子基团)可以稳定 $\mathrm{S}_{\mathrm{N}} \mathrm{Ar}$ 反应中间体 Meisenheimer 复合体, 使得反应容易 进行. 某些含氮杂环如吡啶、二嗪本身是缺电子的, 这 类分子不需要引入额外的活化基团, 即可较容易地通过 $\mathrm{S}_{\mathrm{N}} \mathrm{Ar}$ 实现氟-18 标记. 而在富电子和电中性芳香环通过 $\mathrm{S}_{\mathrm{N}} \mathrm{Ar}$ 进行氟-18 标记十分困难, 这极大地限制了底物的 范围, 增加了放射性药物的设计难度. 为了解决这一难 题, 发现具有优秀活化基团和离去基团的标记反应前体 类型成为人们工作的热点.

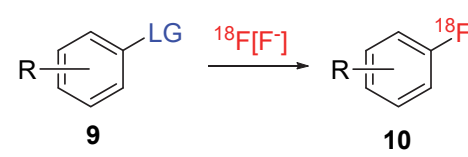

$\mathrm{R}=\mathrm{NO}_{2}, \mathrm{CN}, \mathrm{CF}_{3}$, etc.

$L G=N^{+} R_{3}, N_{2}, I, B r$, OTs, OTf, etc.

\section{1 以酚为前体的脱氧氟代氟-18 标记}

在有机合成中, 酚是常见的构建单元, 以酚为氟18 标记前体进行研究具有广泛前景, 但羟基并不属于 吸电子基团，应用传统的 $\mathrm{S}_{\mathrm{N}} \mathrm{Ar}$ 方式直接构建 $\mathrm{C}\left(\mathrm{sp}^{2}\right)$ ${ }^{18} \mathrm{~F}$ 键十分困难. 2016 年, Ritter 和 Hooker 等 ${ }^{[7]}$ 报道了一 种苯酚类结构脱氧氟化的氟-18 标记方法. 以往 $\mathrm{S}_{\mathrm{N}} \mathrm{Ar}$ 都 是通过形成 Meisenheimer 复合体来进行的，但是该反应 是一种通过形成脲阳离子中间体的协同亲核芳香取代 反应 $\left(\mathrm{CS}_{\mathrm{N}} \mathrm{Ar}\right.$, concerted nucleophilic aromatic substitution reaction) (Scheme 1).

缺电子的酚可以以较高放射化学转化率(radiochemical conversions, RCC)得到标记产物，而富电子酚的 平衡常数 $K_{1}$ 更小, 在过渡态发生协同脱氧氟化之前氟 就从中间体中脱除并分解, 进而导致富电型酚的放射化 学转化率下降. 此反应无需严格的无水无氧环境, 都可 以耐受包括胺、酚、硫梄和酰胺在内的多种官能团, 在 芳香环和杂环都可以进行放射性脱氧氟化, 并且具有很 高的放射化学转化率; 此外还标记了 mGluR5 的显像剂 FPEB (Eq. 2).

为了解决富电性酚低放射性转化率的问题, 2017 年 Ritter 和 Hooker 等 ${ }^{[8]}$ 对此方法进行了深入研究. 他们发 
<smiles>[R][R]c1cccc(OC2([18F])N([Al])C=CN2[Al])c1[R]</smiles>

12

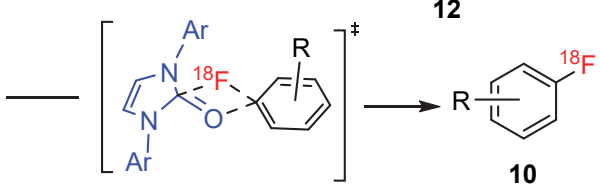

$\Delta \mathrm{G}^{\neq}=91.1 \mathrm{~kJ} \cdot \mathrm{mol}^{-1}$

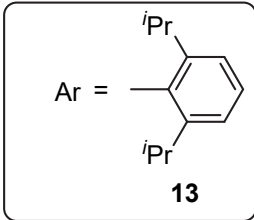

图式 1 以酚为前体的脱氧氟代氟-18 标记

Scheme $1 \quad\left[{ }^{18} \mathrm{~F}\right]$ Deoxyfluorination of phenols

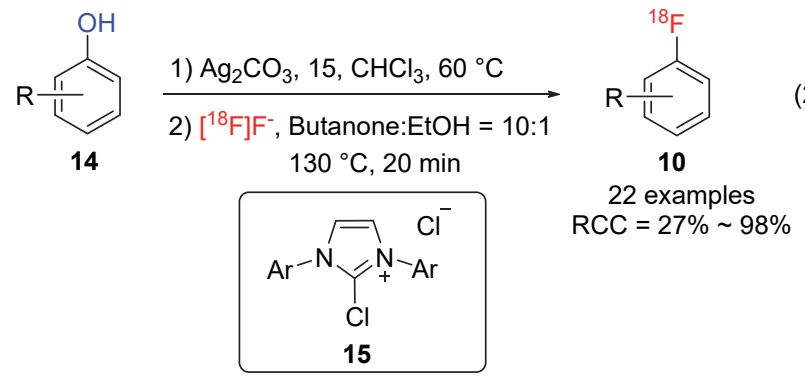<smiles>Cc1cncc(F)c1</smiles>

16

$\mathrm{RCC}=75 \%$<smiles>CCOCc1nc([18F])c2c(C)c(C(=O)CC)sc2n1</smiles>

19

$\mathrm{RCC}=81 \%$

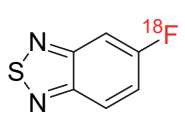

17

$\mathrm{RCC}=27 \%$

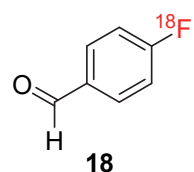

$\mathrm{RCC}=99 \%$

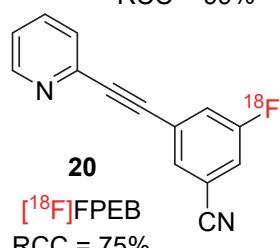

现了一种具有双配体的钉催化剂, 从而实现了富电子苯 酚的脱氧氟-18 标记. 如上文所述, 富电性的酚在形成 $\mathrm{CS}_{\mathrm{N}} \mathrm{Ar}$ 过渡态中间体并进一步转化时, 平衡常数 $K_{1}$ 较 小, 使得仅有少量的中间体脱氧氟代形成目标标记产 物. 而引入具有双配体得钉催化剂后形成了 $\eta^{6}-\pi$ 过渡 态，富电性酚芳环上的电子以此向钉侧转移，使得平衡 常数 $K_{2}>>K_{1}$, 有利于过渡态脱氧氟-18 标记成为目标 化合物(Scheme 2).

这一方法将底物范围扩大至最富电子的苯酚, 降低 了对于底物电性的要求, 芐胺类底物也得到了中等的放 射化学转化率(non-decay-corrected radiochemical yield,

$\mathrm{RCY})$ ，同时也成功标记了左旋多巴及脱氧雌酚酮．反 应操作简便且可以在有水分的空气中进行，同时实现了 自动化合成(Eq. 3).<smiles></smiles>

21
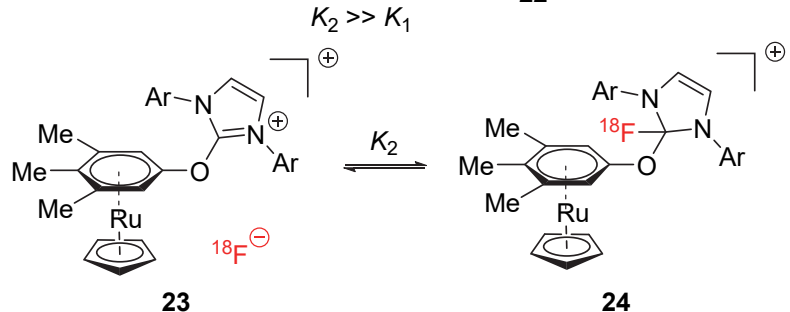

图式 2 钉催化酚的氧氟代氟-18 标记

Scheme 2 Ruthenium-mediated $\left[{ }^{18} \mathrm{~F}\right]$ deoxyfluorination of phenols
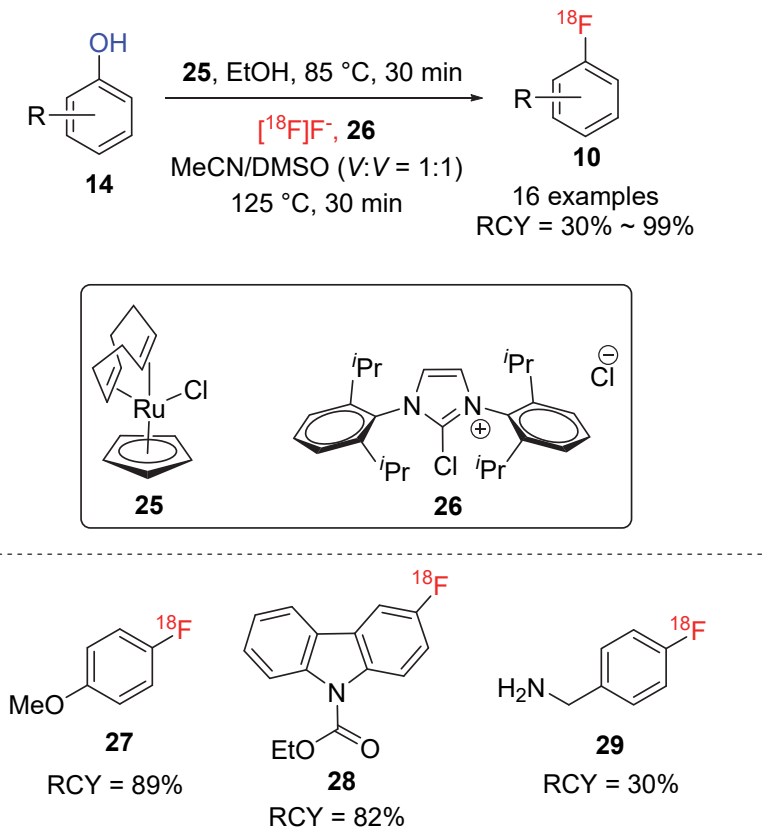<smiles>COC(=O)NC(Cc1ccc(F)cc1)C(=O)OC</smiles>

30

$\mathrm{RCY}=99 \%$

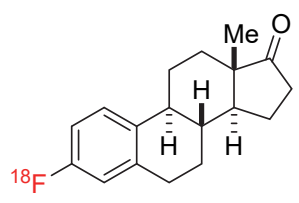

31 $\mathrm{RCY}=88 \%$

\section{2 以酚为前体的氧化氟-18 标记}

2012 年 Gouverneur 课题组 ${ }^{[9]}$ 报道了一种在苯酚羟 基对位发生的原位氟-18 标记反应. 这一反应分为两步, 首先是 4-叔丁基酚在氧化剂二乙酰氧基碘苯(PIDA)和 
亲核氟-18 氟源存在条件下生成 4- $\left[{ }^{18} \mathrm{~F}\right]$ 氟-4-叔丁基-2,5环已烯-1-酮, 随后加酸酸化后脱去异丁烯, 再芳构化得 到对位标记的氟-18 苯酚, 这两步通过一锅法完成 (Scheme 3). 该方法可直接用于未保护的酚羟基前体的 标记, 并且在反应后仍然保留羟基这一重要官能团, 反 应也无需加入金属催化剂及结构复杂的配体, 这大大降 低了后处理的困难程度, 并增加了反应的适用范围.
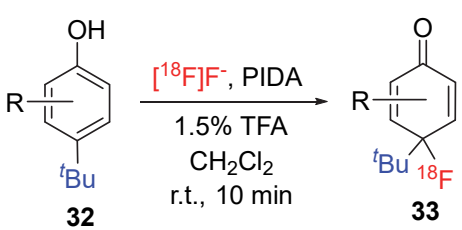

33

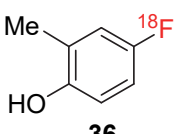

36 $\underset{\text { r.t., } 10 \mathrm{~min}}{\stackrel{10 \% \mathrm{TFA}}{\mathrm{N}}}$

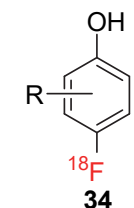

8 examples $\mathrm{RCY}=11 \% \sim 32 \%$

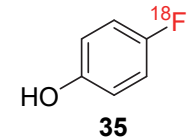

35
$\mathrm{RCY}=15 \%(n=8)$

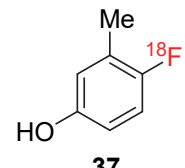

37<smiles>Oc1ccc(Br)cc1Br</smiles>

38

$\mathrm{RCY}=16 \%(n=11)$<smiles>O=C(c1ccccc1)c1cc(Br)ccc1O</smiles>

39

图式 3 以酚为前体的氧化氟代氟-18 标记

Scheme 3 Oxidative $\left[{ }^{18} \mathrm{~F}\right]$ fluorination of phenols

\section{3 以芳基硫化合物为前体进行氟-18 标记}

近年来, 人们在开发新型芳基硫前体方面做了大量 的研究工作, 其中最重要的是二芳基亚砜和三芳基硫鎓 盐前体. 2012 年, Lehmann 和 Dinkelborg 等 ${ }^{[10]}$ 报道了三 芳基硫鎓盐可作为氟-18 标记反应的前体, ${ }^{18} \mathrm{~F}$-氟化优先 发生在相对缺电子的芳香环上, 得到相应的氟-18 标记 的芳香化合物, 余下部分则作为二芳基硫醚离开. 不同 的溶剂、碱及反应温度对产物的转化率及副产物的产量 有很大影响, 该方法适用于包括酰胺在内的一系列取代 的芳香体系, 特别是以较高的放射化学产率标记了肽 段，显示这一方法具有潜在标记肽的前景(Eq. 4).

药物中通常含有路易斯碱基团, 为了扩大三芳基硫 鎓盐这类前体在放射药物中的应用, 2015 年, Lythgoe 和 Årstad 等 ${ }^{[11]}$ 改造了待标记芳环以外的另外两个芳环, 通 常是在邻对位引入甲氧基等给电子基团, 显著提高了放 射化学收率, 并将底物范围扩大到富电芳环和电中性芳 环. 该反应兼容脂肪族胺、亚胺和碱性杂环, 以及醇、 醛、酮和酯这类常见结构(Eq. 5).

2013 年, Pike 课题组 ${ }^{[12]}$ 发现许多二芳基亚砜可以很 容易地与 ${ }^{18} \mathrm{~F}$ 氟离子反应, 生成高放射化学收率的氟- 18 标记的芳香化合物. 当亚砜两侧两个芳香基团相同时,
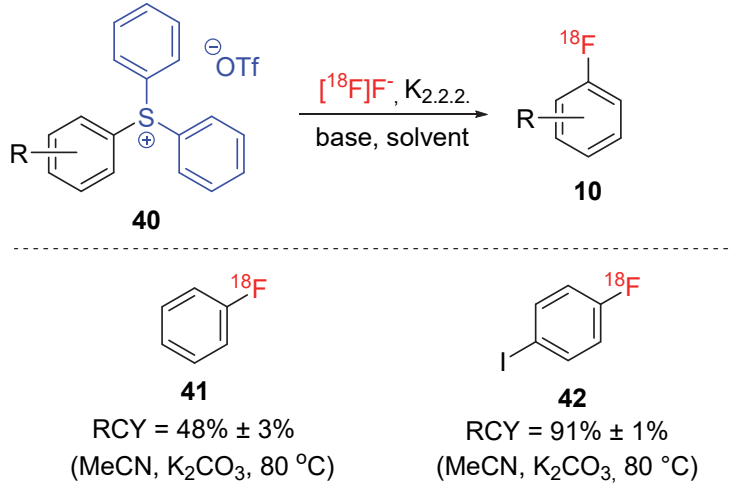<smiles>CC(C)[C@H](NC(=O)c1ccc(F)cc1)C(=O)NCCC(=O)N[C@@H](Cc1ccccc1)C(=O)NCC(N)=O</smiles>

$\mathrm{RCY}=68 \% \pm 4 \%$ $\left(\mathrm{MeCN}, \mathrm{Cs}_{2} \mathrm{CO}_{3}, 80^{\circ} \mathrm{C}\right)$<smiles>O=C(B(c1ccccc1)c1ccccc1)c1ccccc1</smiles>

$\mathrm{RCY}=13 \% \pm 2 \%,\left(150^{\circ} \mathrm{C}\right.$ $\left.\mathrm{R}^{1}=\mathrm{R}^{2}=4-\mathrm{OCH}_{3}\right)(n=3)$

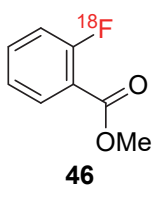
$\left.\mathrm{R}^{1}=4-\mathrm{OCH}_{3}, \mathrm{R}^{2}=\mathrm{H}\right)(n=3)$
$\mathrm{RCY}=64 \% \pm 1 \%\left(110{ }^{\circ} \mathrm{C}\right.$,
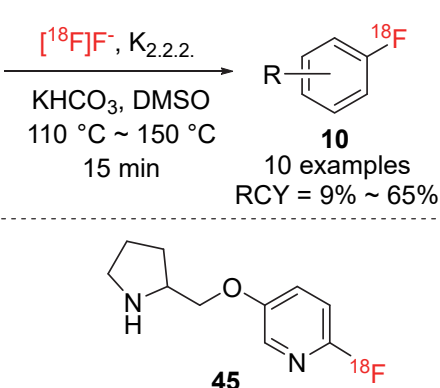

$\mathrm{RCY}=51 \% \pm 5 \%$

$\left(110{ }^{\circ} \mathrm{C}, \mathrm{R}^{1}=\mathrm{R}^{2}=\mathrm{H}\right)$ $(n=3)$

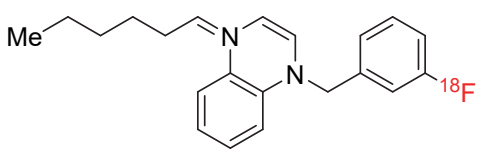

47

$\mathrm{RCY}=30 \% \pm 6 \%\left(120{ }^{\circ} \mathrm{C}\right.$, $\left.\mathrm{R}^{1}=\mathrm{R}^{2}=4-\mathrm{OCH}_{3}\right)(n=3)$
缺电子型芳环使用该方法能以很好的放射化学收率得 到标记产物; 当亚砜不对称时, 相对缺电子芳环也能以 高放射化学收率得到高度选择性的标记产物(Eq. 6).

\section{4 以芳基高价磑化合物为前体进行氟-18 标记}

高价芳基碘化合物是芳香亲核取代反应的优秀底 物，放射化学家一直致力于应用这类化合物作为氟-18 标记的前体，其中已报道的可用于氟-18 标记的前体类 型主要有二芳基碘鈆盐，碘叶立德，二乙酰氧基碘苯. 1995 年 Pike 和 Aigbirhio 等 ${ }^{[13]}$ 首次把此类前体与亲核氟 源联用研究芳香环氟-18 标记. 芳环上的电性差异极大 得影响了氟-18 标记的选择性和放射化学收率, 富电子 芳环几乎不能得到标记产物(Eq. 7). 
<smiles>[R][R]1ccc(S(=O)c2ccccc2)cc1</smiles>

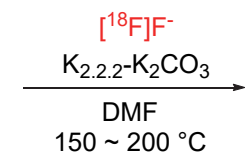

48 $188 \sim 251 \mathrm{~s}$

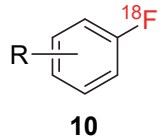

16 examples $\mathrm{RCY}=2 \% \sim 94 \%$<smiles>O=[N+]([O-])c1ccc(Br)cc1</smiles>

49

$\mathrm{RCY}=91 \%$ $\left(\mathrm{R}=\mathrm{R}^{1}=4-\mathrm{NO}_{2}\right)$ $\left(\mathrm{R}=\mathrm{R}^{1}=3-\mathrm{NO}_{2}\right)$<smiles>O=[N+]([O-])c1ccc(Br)cc1</smiles>

$49 \mathrm{RCY}=93 \%$ $\left(\mathrm{R}=4-\mathrm{NO}_{2}\right.$, $\mathrm{R}^{1}=4-\mathrm{CN}$ )<smiles>Cc1cc([N+](=O)[O-])ccc1Br</smiles>

$52 \mathrm{RCY}=73 \%$ ( $\mathrm{R}=2-\mathrm{Me}-4-\mathrm{NO}_{2}$, $\mathrm{R}^{1}=\mathrm{H}$ )

51 $\mathrm{RCY}=82 \%$ $\left(R=R^{1}=4-C N\right)$

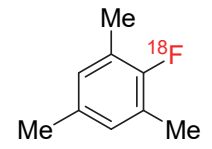

$53 \mathrm{RCY}=61 \%$

$\left(\mathrm{R}=2,6-\mathrm{Me}_{2}-4-\mathrm{NO}_{2}\right.$, $\mathrm{R}^{1}=\mathrm{H}$ )<smiles>N#Cc1ccc(Br)cc1</smiles>

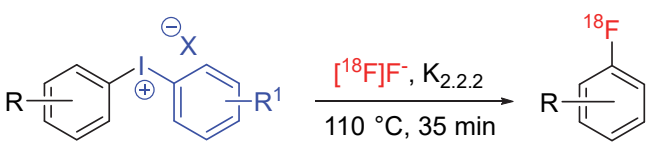

48

1012 examples $\mathrm{RCY}=7.5 \% \sim 88 \%$

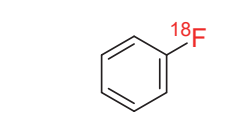

$41 \mathrm{RCY}=31 \%$ $\left(\mathrm{R}=\mathrm{R}^{1}=\mathrm{H}, \mathrm{X}^{-}=\mathrm{Cl}^{-}\right)$

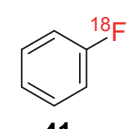

41

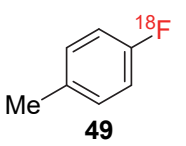

$\mathrm{RCY}=10 \%$

$41: 49=72 \%: 28 \%$

$\left(\mathrm{R}=\mathrm{H}, \mathrm{R}^{1}=4-\mathrm{Me}, \mathrm{X}^{-}=\mathrm{CF}_{3} \mathrm{SO}_{3}^{-}\right)$

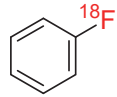

$41 \mathrm{RCY}=15 \%$ $\left(\mathrm{R}=\mathrm{R}^{1}=\mathrm{H}, \mathrm{X}^{-}=\mathrm{CF}_{3} \mathrm{SO}_{3}{ }^{-}\right)$<smiles>Fc1ccccc1</smiles>

41<smiles>COc1ccc(Br)cc1</smiles>

27

$$
\begin{gathered}
\mathrm{RCY}=35 \% \\
\mathbf{4 1}: \mathbf{2 7}=100 \%: 0 \% \\
\left(\mathrm{R}=\mathrm{H}, \mathrm{R}^{1}=4-\mathrm{OMe}, \mathrm{X}^{-}=\mathrm{Br}^{-}\right)
\end{gathered}
$$

为实现二芳基碘鎓盐两个芳环氟-18 标记时的选择 性, 2007 年, Coenen 课题组 ${ }^{[14]}$ 首次把富电性的噻吩引入 二芳基碘鎓盐前体中. 2 -噻吩使得 ${ }^{18} \mathrm{~F}$ 氟离子高度区域选 择地对碘鎓盐另一端的碳原子进攻，得到没有其他放射 性副产物的高纯目标标记产物. 此外, 溴化碘鎓盐在溶 液中的溶解性超过相应的碘化盐、三氟甲磺酸盐和对甲 苯磺酸盐, 因而具有最好的标记效果; 对取代基位置的 研究则表明, 邻位效应很大程度上影响了反应的发生, 邻位取代的芳环是这类反应理想的底物(Eq. 8).

2010 年 Pike 课题组 ${ }^{[15]}$ 使用微反应器技术进一步研 究二芳基碘鎓盐氟-18 标记过程中的邻位效应, 并对各 苯环邻位取代基产生邻位效应的能力进行了排序, 典型 的顺序如下: $2,6-\mathrm{Me}_{2}>2,4,6-\mathrm{Me}_{3}>\mathrm{Br}>\mathrm{Me}>\mathrm{Et} \approx{ }^{i} \mathrm{Pr}>$
$\mathrm{H}>\mathrm{OMe}$ ，这表明邻位取代基产生的邻位效应不仅仅取 决于取代基的体积. ${ }^{18} \mathrm{~F}$ 氟离子与具有邻位取代基的二 芳基碘鎓盐可以以极快的速率生成高放射化学收率的 氟-18 标记的芳环, 不对称的二芳基碘鎓盐的 ${ }^{18} \mathrm{~F}$ 标记反 应选择性符合 Curtin-Hammett 原理(Eq. 9).
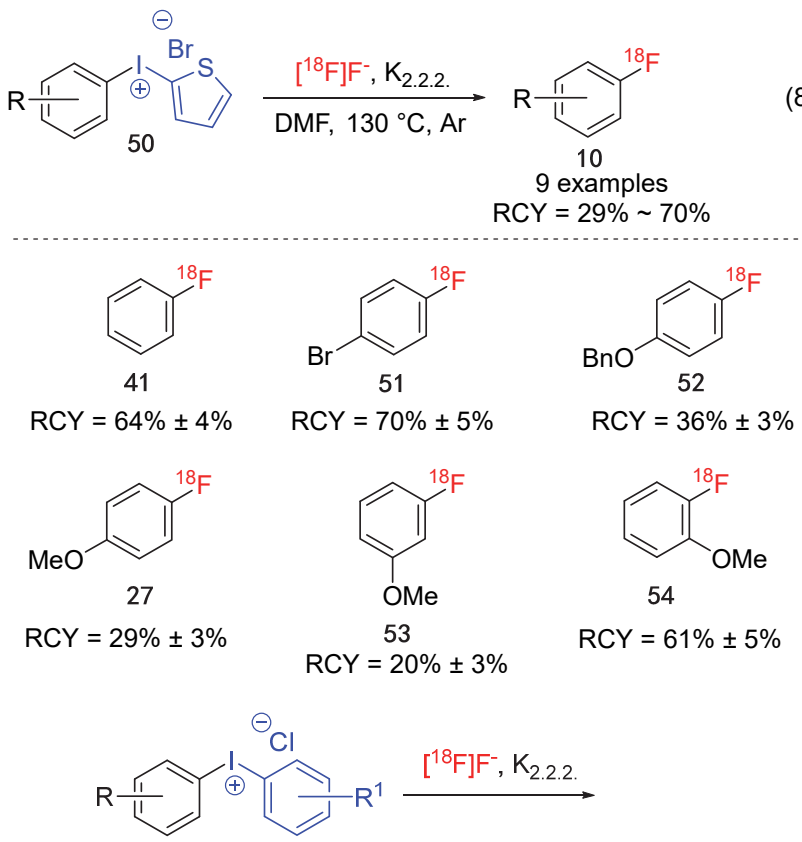

51

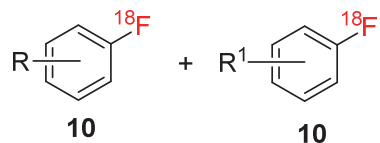<smiles>O=[N+]([O-])c1ccccc1[F+]</smiles>

52

$52 \mathrm{RCY}=57 \%$

$41 \mathrm{RCY}=25 \%$

$\left(110^{\circ} \mathrm{C}, 188 \mathrm{~s}\right.$

DMF- $0.25 \%$ water) $\overbrace{}^{18 F}$

52
$52 \mathrm{RCY}=75 \%$

$53 \mathrm{RCY}=4 \%$

$\left(140{ }^{\circ} \mathrm{C}, 188 \mathrm{~s}\right.$

DMF-0.25\% water)<smiles>COc1ccccc1Br</smiles>

53

41

$41 \mathrm{RCY}=60 \%$ $53 \mathrm{RCY}=6.5 \%$ $\left(140{ }^{\circ} \mathrm{C}, 188 \mathrm{~s}\right.$ DMF- $0.25 \%$ water)<smiles>Cc1ccccc1F</smiles>

54

52

$54 \mathrm{RCY}=48 \%$ $52 \mathrm{RCY}=38 \%$ $\left(170{ }^{\circ} \mathrm{C}, 236 \mathrm{~s}\right.$ anhydrous DMF)
从反应历程分析, 最初的两构象可以快速转化, 而 相应的过渡态 $\mathrm{TS}_{1}$ 和 $\mathrm{TS}_{2}$ 则产生了不同的标记产物. 经 计算，能量更高的过渡态不易形成，这使得反应几乎完 全沿着那条能量更低的过渡态路径进行，最终得到了高 位置选择性的标记产物. 这一发现可用于指导放射性药 物前体的合成(Scheme 4)

2016 年刘国生和梁欢课题组 ${ }^{[16]}$ 报道了一种新型的 

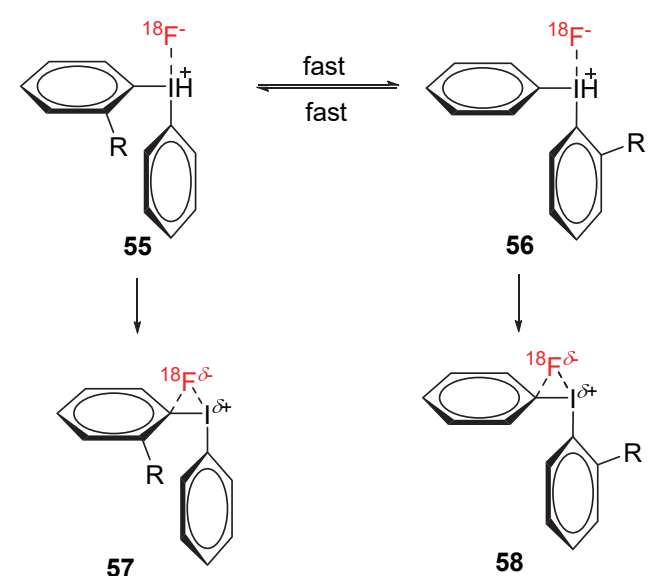

$\mathrm{TS}_{1}$
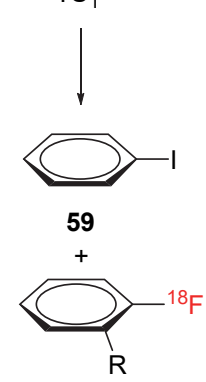

$61 \mathrm{P}_{1}$

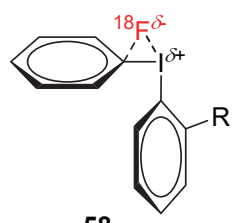

58

$\mathrm{TS}_{2}$
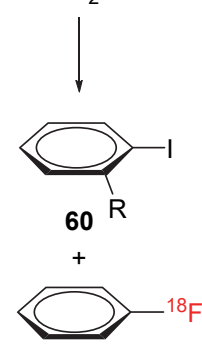

$41 \mathrm{P}_{2}$

图式 4 二芳基碘鎓盐氟-18 标记反应的反应选择性及和机理

Scheme 4 Selectivity and mechanism in $\left[{ }^{18} \mathrm{~F}\right]$ fluorination of aryl iodonium salts

简便高效合成 ${ }^{18} \mathrm{~F}$ 标记的异喹啉的方法. 该反应由两步 组成, 首先是过渡金属银参与的炔烃胺化, 以及随后苯 基异喹啉基碘鎓盐的 ${ }^{18} \mathrm{~F}$ 标记过程, 各种结构的底物耐 受性好, 且放射化学产率高(Eq. 10).

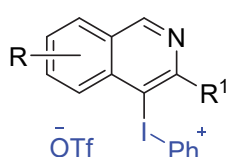

62
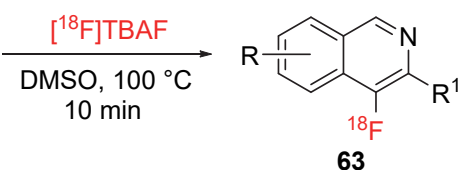

63

22 examples

$\mathrm{RCY}=10 \% \sim 92 \%$<smiles>Cc1ccc2cnc(C(C)(C)C)c(F)c2c1</smiles><smiles>[18F]c1c(-c2ccccc2)ncc2ccccc12</smiles><smiles>[18F]c1c(-c2ccccc2)ncc2sccc12</smiles>

$65 \mathrm{RCY}=26 \% \pm 2 \%$

$67 \mathrm{RCY}=82 \%$

2013 年 Sanford 等及 Scott 课题组 ${ }^{[17]}$ 报道了均三甲 基苯基芳基碘鎓盐用前体的氟-18 标记反应及相应机理. 这一不对称碘鎓盐标记产物的选择性与邻位效应相反, ${ }^{18} \mathrm{~F}$ 氟未标记在双邻位甲基的均三甲苯环上, 而是发生

在另一芳环上; 反应使用过渡金属铜作为催化剂，大大 降低了反应温度, 减少了高温下碘两端芳环过渡态下的 交换速率，降低了副产物的产生，实现了对于富电型、 电中性和缺电型底物的放射性氟标记(Eq. 11).
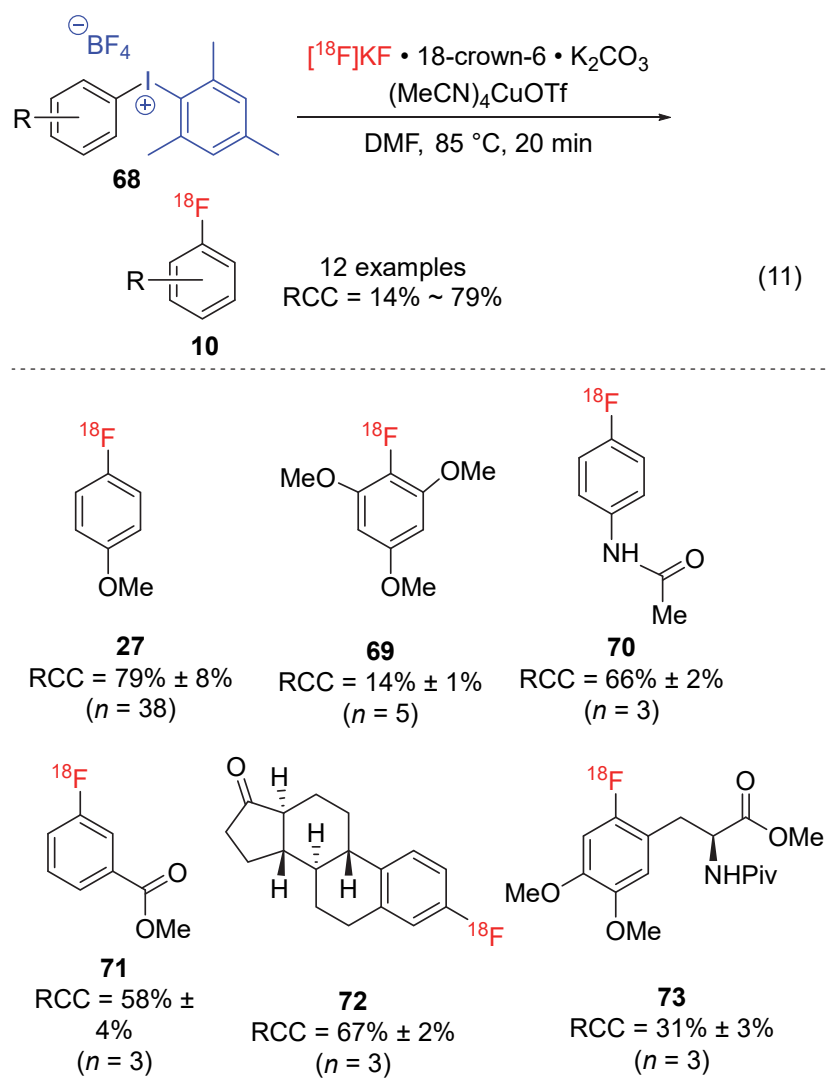

作者提出该反应可能是通过 $\mathrm{Cu}^{\mathrm{I}}$ 和 $\mathrm{Cu}^{\mathrm{III}}$ 催化循环实 现的. 首先是二价铜 74 通过歧化作用或者被溶剂还原 生成具有催化活性的 $\mathrm{Cu}^{\mathrm{I}}$ 物种 $\mathbf{7 5}$, 随后发生阴离子交换 生成单 ${ }^{18} \mathrm{~F}$-取代的中间体 76, 随后被标记前体二芳基碘 鎓盐 77 氧化生成 ${ }^{18} \mathrm{~F}^{-}-\mathrm{Cu}^{\mathrm{III}}$-芳基复合物 79, 最后经由 $\pi$ 络合物 80 发生还原消除, 得到被标记化合物 $41, \mathrm{Cu}^{\mathrm{III}}$ 再 生为 $\mathrm{Cu}^{\mathrm{I}}$ 物种 75 (Scheme 5).

2017 年他们 ${ }^{[18]}$ 对上述方法进行了改进, 通过一锅 法实现富电子芳香环 $\mathrm{C}-\mathrm{H}$ 键的直接氟-18 标记. 这一 方法不需要预先合成稳定性差的均三甲基苯基芳基碘 鎓盐前体，可以直接以稳定的芳香族化合物为原料进行 放射性氟标记(Eq. 12).

芳环上被供电子基团和吸电子基团取代的苯基碘 叶立德可作为芳香亲核取代反应的前体，含有辅基的碘 叶立德性质稳定, $\mathrm{S}_{\mathrm{N}} \mathrm{Ar}$ 反应中可被亲核基团如卤离子取 代，生成相应的卤代基衍生物，因而芳基碘叶立德也是 一种潜在的氟-18 标记底物. 2014 年 Humpert 和 Coenen 等 ${ }^{[19]}$ 报道了芳基碘叶立德作为前体的 ${ }^{18} \mathrm{~F}$ 标记方法. 以 丙二酸环(亚)异丙酯作为辅基、芳环对位引入烷氧基的 


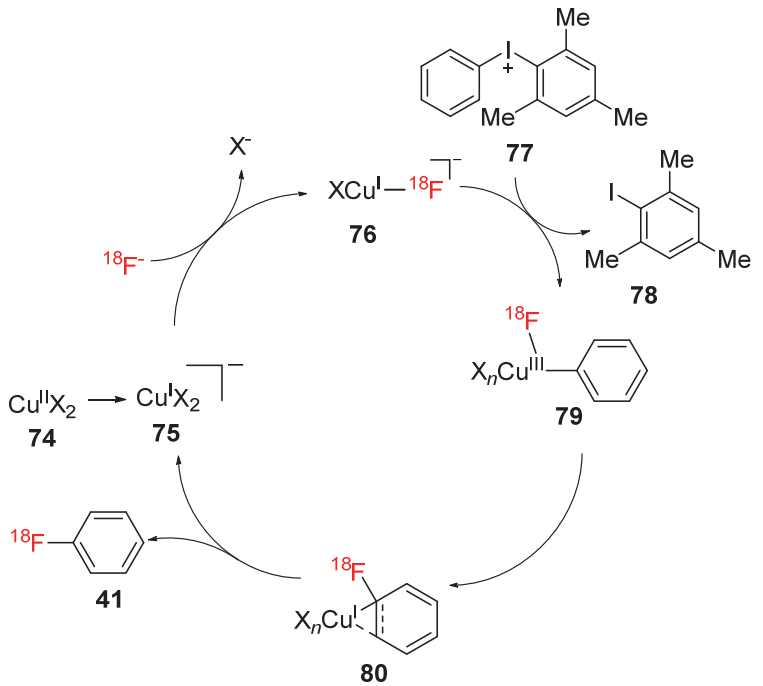

图式 5 铜催化二芳基碘鎓盐氟-18 标记反应可能的反应机理 Scheme 5 Possible mechanism of copper-catalyzed $\left[{ }^{18} \mathrm{~F}\right]$ fluorination of diaryliodonium salts

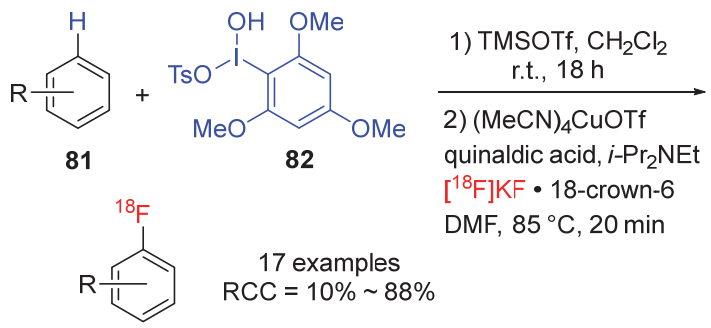

10

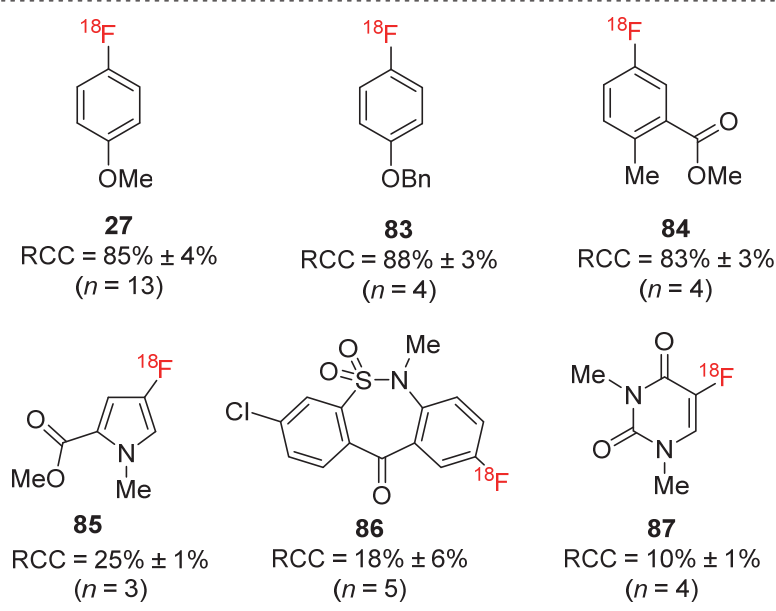

这类芳基碘化合物稳定性也远高于相应的含二芳基碘 鎓盐结构的前体, 适用于富电子和未活化的芳香化合物 的 ${ }^{18} \mathrm{~F}$ 标记, 氟标记发生在原位或邻位, 并且原位的标 记反应更易发生(Eq. 13).

为了降低碘叶立德标记反应中邻位副产物的比例, 2014 年 Vasdev 和 Liang 等 ${ }^{[20]}$ 芳基碘叶立德前体的不同 辅基进行了研究, 试图发现一种能更好稳定碘叶立德中 心碘原子、减少三价碘歧化和分解的辅基. 结果表明不 同结构的辅基放射化学产率略有差异, 其中氧杂环优于

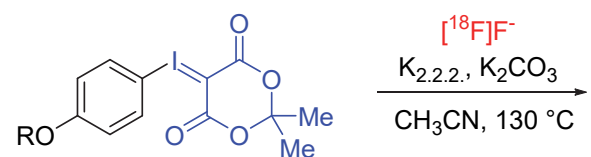

88<smiles>[R]Oc1ccc([18F])cc1</smiles>

89 90<smiles>COc1ccc([18F])cc1</smiles>

$27 \mathrm{RCY}=12 \% \quad 91 \mathrm{RCY}=4 \%$

$83 \mathrm{RCY}=20 \% \quad 92 \mathrm{RCY}=11 \%$

Boc<smiles>CN1CCC(C(Oc2cccc([18F])c2)c2ccccc2)CC1</smiles>

93 $3-\left[{ }^{18} \mathrm{~F}\right] \mathrm{FPPMP}$ $\mathrm{RCY}=10 \%$

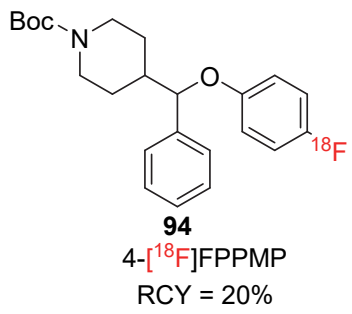

氮杂环，氧杂螺 [6.5]烷又优于螺 [6.6]烷和螺[6.4]烷，含 二氧杂螺 [4.5]癸烷类结构的辅基效果最好(Eq. 14).

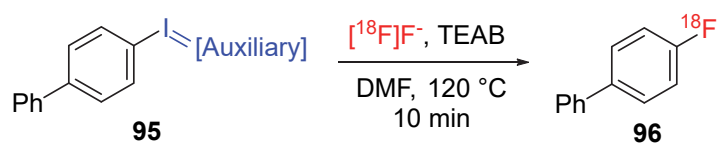<smiles>O=C1NC(=O)C(=O)NC1=O</smiles>

[Auxiliary]

$\mathrm{RCY}=0 \%(n=3)$

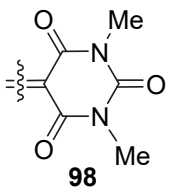

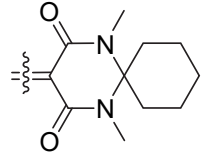

99

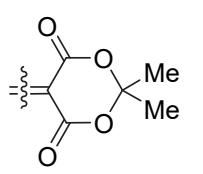

100

$\mathrm{RCY}=47 \%$

$(n=3)$<smiles>O=C(O)C1C(=O)OC2(CCCC2)OC1=O</smiles>

101<smiles>CC1C(=O)OC2(CCC2)OC1=O</smiles>

102 $(n=3)$<smiles>O=C1OC2(CCC2)OC(=O)C1=O</smiles>

103
对多个底物的标记结果表明，这一反应能够直接和 高位置选择性地在原位放射性标记非活性芳烃和空间 受阻芳烃，并兼容酯类、氨基甲酸酯类、卤化物类、酰 胺类、硝基、三氟甲基、酮类和杂环等各类底物(Eq. 15).

同时他们也研究了反应机理，可能的反应历程如 下: ${ }^{18} \mathrm{~F}$ 氟离子首先插入到碘与辅基的双键中形成三角 双雉中间态，两种构象 110 和 111 可互相转化，但仅当 ${ }^{18} \mathrm{~F}$ 氟离子与辅基处于轴向时的构象 111 才可发生消除 反应得到氟标记产物(Scheme 6). 


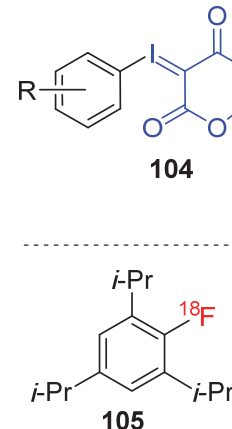

$\mathrm{RCY}=56 \% \pm 6 \%$

$(n=3)$<smiles>Brc1cccnc1</smiles>

108

$\mathrm{RCY}=65 \% \pm 11 \%$ $(n=3)$
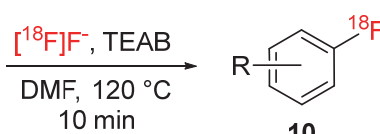

10

17 examples

$\mathrm{RCY}=7 \% \sim 77 \%$

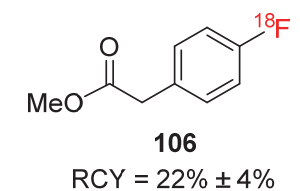

$(n=3)$

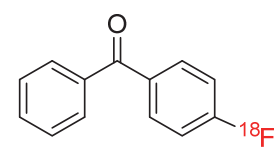

109

$\mathrm{RCY}=55 \% \pm 8 \%$ $(n=3)$
$\mathrm{RCY}=22 \% \pm 4 \%$

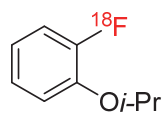

107

$\mathrm{RCY}=16 \% \pm 3 \%$ $(n=3)$<smiles>COC(=O)c1cccc(Br)c1</smiles>

72

$\mathrm{RCY}=77 \% \pm 7 \%$ $(n=3)$
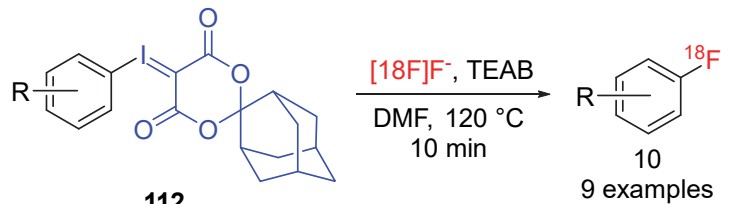

(16) $\mathrm{RCY}=25 \% \sim 82 \%$

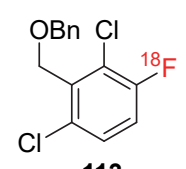

113

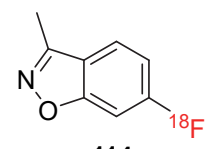

114

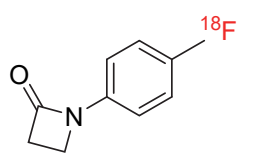

115
$\mathrm{RCY}=82 \% \pm 6 \% \quad \mathrm{RCY}=25 \% \pm 4 \% \quad \mathrm{RCY}=71 \% \pm 13 \%$<smiles>O=C(O)N1CCCC(COc2ccc(Br)cn2)C1</smiles>

$\mathrm{RCY}=45 \% \pm 4 \%$<smiles>CC(C)(C)OC(=O)N(C(=O)OC(C)(C)C)c1ccc(Cl)c(OCc2cccc([18F])c2)c1</smiles>

117
一步提高了标记效率. 带有吸电子基的底物本身氧化电 势较高, 加入大体积配体后阻碍了氟化优势构象中间体 的形成，使得放射化学转化率反而下降(Eq. 17)..
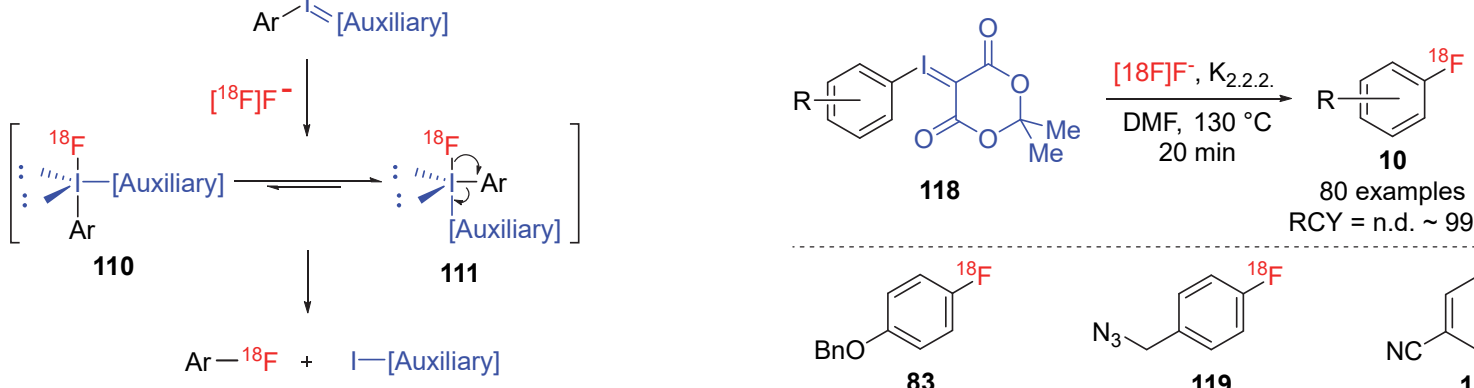

图式 6 碘叶立德氟-18 标记反应可能的反应机理

Scheme 6 Possible mechanism of $\left[{ }^{18} \mathrm{~F}\right]$ fluorination of aryl iodonium ylides

2016 年该研究团队 ${ }^{[21]}$ 又报道了一种新型辅基 SPIAd (spiroadamantyl-1,3-dioxane-4,6-dione); 该辅基是 金刚烷结构类似物，具有很强的空间位阻效应，含有这 种辅基前体的稳定性优于之前报道的前体, 因此非常有 利于未活化和富含电子的底物的 ${ }^{18} \mathrm{~F}$ 标记, 用此方法对 放射性药物常见构建单元进行标记, 均取得了满意的放 射化学收率(Eq. 16).

2017 年 Riss 课题组 ${ }^{[22]}$ 仍以丙二酸环(亚)异丙酯作 为辅基, 通过加入三苯膦进一步优化了标记反应. 部分 碘叶立德前体稳定性差, 自身以一级动力学方式快速降 解是影响放射化学收率的决定性原因, 多数前体在被 氟-18 标记前就已经降解. 研究表明, 加入三苯基膦等 大体积的配体后虽几乎不能抑制标记前体自身的降解, 但是可以降低标记反应的活化能, 加快标记反应速率, 从而提高放射化学收率. 在用 $\mathrm{N}_{2}$ 吹出反应体系中的 $\mathrm{O}_{2}$ 后, 抑制了三苯基膦转化为无配体活性的三苯氧磷, 进
80 examples $\mathrm{RCY}=$ n.d. $\sim 99 \%$

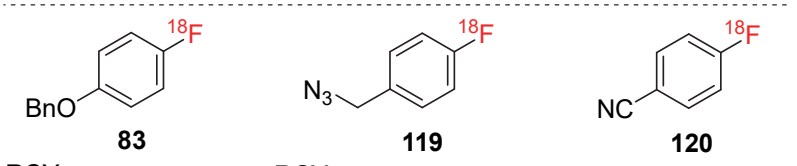

$\mathrm{RCY}=$ $\mathrm{RCY}=$

$-\mathrm{PPh}_{3} /-\mathrm{N}_{2} 46 \% \pm 11 \%-\mathrm{PPh}_{3} /-\mathrm{N}_{2} 33 \% \pm 15 \%$ $+\mathrm{PPh}_{3} /-\mathrm{N}_{2} 65 \% \pm 4 \%+\mathrm{PPh}_{3} /-\mathrm{N}_{2}$ n.d. $-\mathrm{PPh}_{3} /+\mathrm{N}_{2} 75 \% \pm 2 \%-\mathrm{PPh}_{3} /+\mathrm{N}_{2} 92 \% \pm 2 \%$ $+\mathrm{PPh}_{3} /+\mathrm{N}_{2} 75 \% \pm 2 \%+\mathrm{PPh}_{3} /+\mathrm{N}_{2}$ n.d.

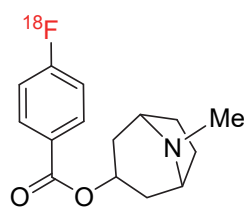

121

$\mathrm{RCY}=$

$-\mathrm{PPh}_{3} /-\mathrm{N}_{2} 82 \% \pm 5 \%$ $+\mathrm{PPh}_{3} /-\mathrm{N}_{2} 88 \% \pm 0 \%$ $-\mathrm{PPh}_{3} /+\mathrm{N}_{2} 96 \% \pm 1 \%$ $+\mathrm{PPh}_{3} /+\mathrm{N}_{2} 94 \% \pm 2 \%$

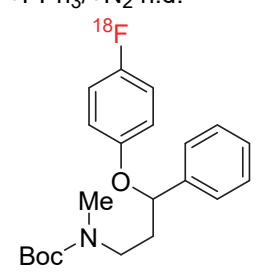

$\mathrm{RCY}=$

$-\mathrm{PPh}_{3} /-\mathrm{N}_{2} 19 \% \pm 7 \%$ $+\mathrm{PPh}_{3} /-\mathrm{N}_{2} 23 \% \pm 8 \%$ $-\mathrm{PPh}_{3} /+\mathrm{N}_{2} 51 \% \pm 6 \%$ $+\mathrm{PPh}_{3} /+\mathrm{N}_{2} 52 \%+7 \%$
RCY =

$-\mathrm{PPh}_{3} /-\mathrm{N}_{2} 91 \% \pm 1 \%$ $+\mathrm{PPh}_{3} /-\mathrm{N}_{2} 79 \% \pm 9 \%$ $-\mathrm{PPh}_{3} /+\mathrm{N}_{2} 90 \% \pm 4 \%$ $+\mathrm{PPh}_{3} /+\mathrm{N}_{2} 67 \% \pm 15 \%$<smiles>CC(C)(C)OC(=O)n1ccc2cc(Br)ccc21</smiles>

123

$\mathrm{RCY}=$

$-\mathrm{PPh}_{3} /-\mathrm{N}_{2} 7 \% \pm 2 \%$ $+\mathrm{PPh}_{3} /-\mathrm{N}_{2} 24 \% \pm 2 \%$ $-\mathrm{PPh}_{3} /+\mathrm{N}_{2} 37 \% \pm 3 \%$ $+\mathrm{PPh}_{3} /+\mathrm{N}_{2} 38 \% \pm 4 \%$
二乙酰氧基碘苯是二芳基碘鎓盐和碘叶立德合成 的上游产品, 若能直接以二乙酰氧基碘苯为氟-18 标记 前体即可减少放射药物整体的合成步骤. 2017 年 Lu 和 Pike 等[23]报道了二乙酰氧基碘基芳香化合物可用于制 备几种 ${ }^{18} \mathrm{~F}$ 标记的芳香化合物, 特别是可用于具有一系 列强吸电子取代基的芳香化合物和在间位含有甲基的 芳香化合物, 具有中等的放射化学转化率(Eq.18). 放射 
化学收率与取代基 Hammett 常数并不呈线性关系, 而且 取代基的邻位效应十分明显. 该方法是新的将取代的碘 芳烃经二乙酰氧基碘苯转化为取代的氟-18 标记芳烃的 两步反应，可用于碘苯类分子的直接氟-18 标记.<smiles>[R][R]1ccccc1I(O[Na])OC(C)=O</smiles>

124

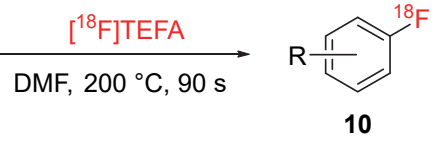

21 examples RCY up to $63 \%$<smiles>Cc1ccc(F)cc1</smiles>

$49 \mathrm{RCY}=20 \%$

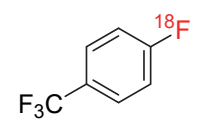

$125 \mathrm{RCY}=51 \%$
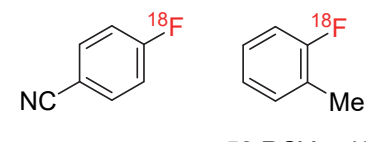<smiles>COc1cccc(F)c1</smiles><smiles>O=[N+]([O-])c1cccc(F)c1</smiles>

$91 \mathrm{RCY}=3 \%$

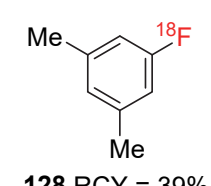

(18)
$120 \mathrm{RCY}=54 \%$<smiles>N#Cc1cccc(F)c1</smiles>

$127 \mathrm{RCY}=9 \%$

\section{5 以芳基硼酸和芳基硼酸酯为前体进行氟-18 标记}

芳基硼酸酯及芳基嗍酸是碳氢键功能化中常见的 构建基团且易通过 Miyaura 嗍酸化反应得到，同时对空 气和水分稳定, 便于长期保存. 2013 年 Sanford 课题 组 ${ }^{[24]}$ 在研究芳基三氟硼酸钾的氟-19 冷标记时发现芳基 嗍酸频哪醇也能取得较好的氟取代效果. 2014 年, Gouverneur 课题组 ${ }^{[25]}$ 将上述方法应用到氟-18 热标记中, 报 道了吡啶络合的铜催化剂参与的芳基嗍酸频哪醇酯的 氟-18 标记反应，芳基硼酸前体对空气和水分稳定，并 且易于获得, 是理想的用于 ${ }^{18} \mathrm{~F}$ 放射性标记的前体. 这 一反应高度依赖 $\mathrm{Cu}(\mathrm{OTf})_{2}(\mathrm{py})_{4}$ (三氟甲磺酸铜四吡啶络 合物)作为催化剂, 空气代替氮气做反应容器填充气时 有助于反应的进行, 显著提高了放射化学收率. 可耐受 大多数电中性、缺电子和富电子型芳香化合物及各种官 能团, 醇和胺裸露的活泼氢被保护后可显著提高放射化 学收率, 在碳碳双键上的标记也取得了中等水平的 RCY (Eq. 19).

这一反应前体易合成和储存、铜催化剂后处理方便 且残留少, 标记方法适用范围且放射化学收率高, 得到 了广泛的应用[26-36]. 此后该课题组又改进了几种常用放 射性药物的标记方法 ${ }^{[37]}$, 并研究了杂环对氟-18 标记放 射化学收率的影响及上百种不同杂环位点上的具体影 响 ${ }^{[38]}$. 某些杂环可能通过促进芳基从嗍到铜的转金属 或从 $\mathrm{Cu}^{\mathrm{III}}{ }^{18} \mathrm{~F}$ 中间体中还原消除来影响 ${ }^{18} \mathrm{~F}$ 氟化, 而有 些具有强 Lewis 碱性配位杂原子的杂环可能通过生成无 活性的铜物种来阻止 ${ }^{18} \mathrm{~F}$ 氟化. 杂环中存在未被保护的 氮原子时，这些杂环可能与芳基硼试剂偶联，同时/或与
$\mathrm{Cu}^{\mathrm{II}}$ 配合物发生强烈配位导致反应不能发生.
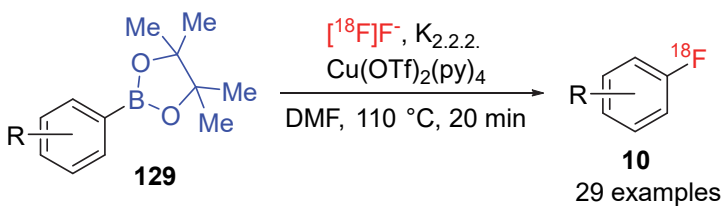

$\mathrm{RCY}=5 \% \sim 74 \%$

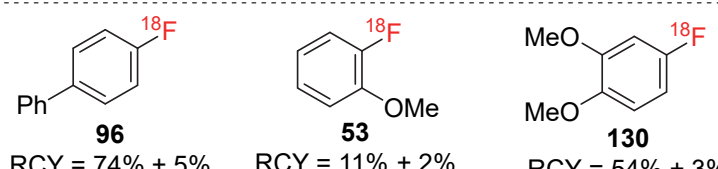

$\mathrm{RCY}=74 \% \pm 5 \% \quad \mathrm{RCY}=11 \% \pm 2 \% \quad \mathrm{RCY}=54 \% \pm 3 \%$

$(n=4) \quad(n=4)$

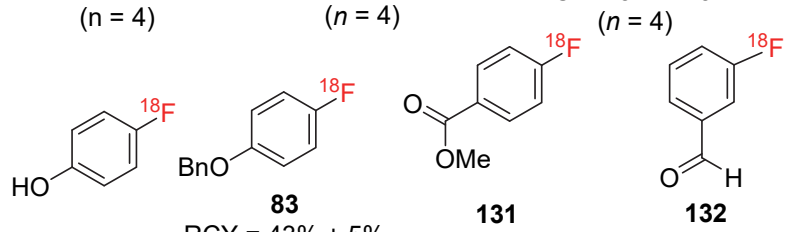

131

132

$\begin{array}{cccc}35 & \mathrm{RCY}=43 \% \pm 5 \% & \mathrm{RCY}=66 \% \pm 6 \% & \mathrm{RCY}=59 \% \pm \\ \mathrm{RCY}=7 \% \pm 2 \% & (n=4) & (n=4) & (n=4)\end{array}$

$(n=4)$

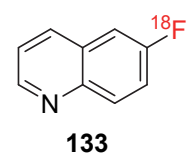

$\mathrm{RCY}=59 \% \pm 8 \%$ $(n=4)$

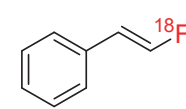

134

$\mathrm{RCY}=26 \% \pm 4 \%$ $(n=4)$<smiles></smiles>

135

$\mathrm{RCY}=58 \% \pm 9 \%$ $(n=4)$
2015 年 Sanford 和 Scott 等 ${ }^{[39]}$ 报道了以芳基硼酸为 前体的 ${ }^{18} \mathrm{~F}$ 标记反应. 催化剂 $\mathrm{Cu}(\mathrm{OTf})_{2}$ 无需预先制成活 性物种 $\mathrm{Cu}(\mathrm{OTf})_{2}(\mathrm{py})_{4}$ ，两者经简单混合即可达到相同的 催化效力，与上述芳基硼酸酯底物应用范围类似，芳基 嘲酸也可兼容多种基团(Eq. 20).

2016 年 Zlatopolskiy 课题组 ${ }^{[40] 、 Z h a n g ~}$ 等 $^{[41]}$ 对上述 芳基硼酸、硼酸频哪醇酯氟-18 标记中的 ${ }^{18} \mathrm{~F}$ 氟离子洗脱 过程进行了改进，分别用 $n-\mathrm{BuOH} / \mathrm{Et}_{4} \mathrm{NHCO}_{3}$ 体系和 DMAP.OTf/DMF 体系代替 $\mathrm{K}_{2} \mathrm{CO}_{3} / \mathrm{K}_{2.2 .2} / \mathrm{ACN} / \mathrm{H}_{2} \mathrm{O}$ 体系 进行洗脱，后继无需常规氟-18 标记中的繁琐共沸干燥 过程，这样减少了这一过程中放射性氟离子的损失，进 一步提高了放射化学收率. 其后又有研究者 ${ }^{[42-43]}$ 用

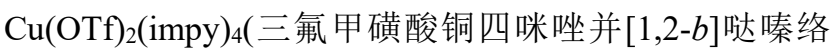
合物)和 DMI (1,3-二甲基-2-咪唑啉酮)分别代替催化剂 $\mathrm{Cu}(\mathrm{OTf})_{2}(\mathrm{py})_{4}$ 和溶剂 DMF 对芳基硼酸频哪醇酯进行氟18 标记, 进一步提高了放射化学收率.

\section{6 以 $N$-芳基悉尼酮为前体进行氟-18 标记}

悉尼酮是一种非常稳定介离子化合物，芳环上取代 的 1,2,3-噁二唑基序列的悉尼酮基团具有吸电子性质, 这一特点使得悉尼酮有可能成为芳香氟-18 标记前体. 2017 年 Murphy 课题组 ${ }^{[44]}$ 报道了这一成果. 该方法以芳 香族氨基化合物为起始，经过两步反应得到芳基悉尼酮 结构; 随后在 ${ }^{18} \mathrm{~F}$ 氟离子的作用下得到被标记的产物. 

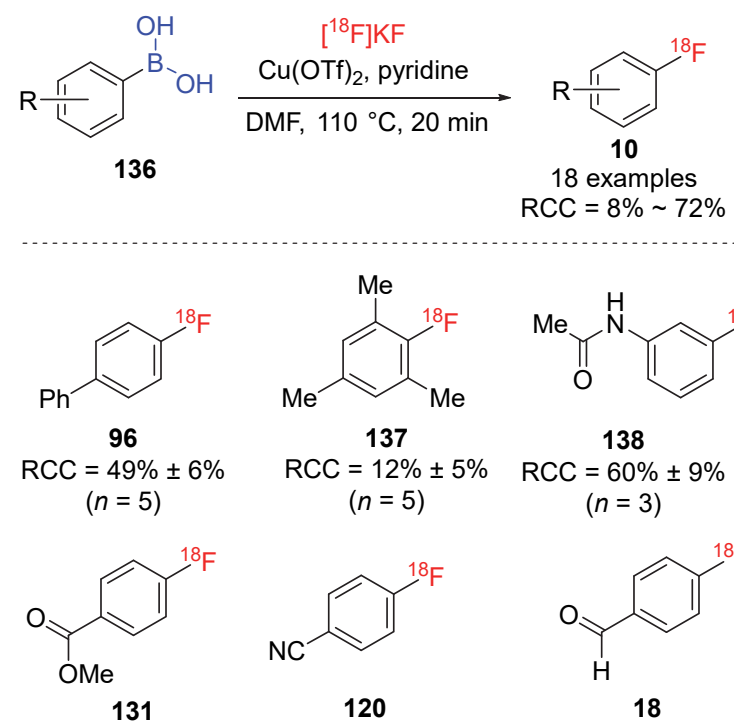

$\mathrm{RCC}=49 \% \pm 9 \%$ RCC $=47 \% \pm 11 \%$ $(n=3)$

$$
(n=4)
$$

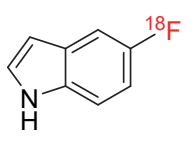

139

$(n=5)$

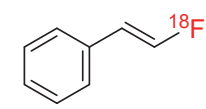

134

$=73 \% \pm 9 \%$ $(n=4)$
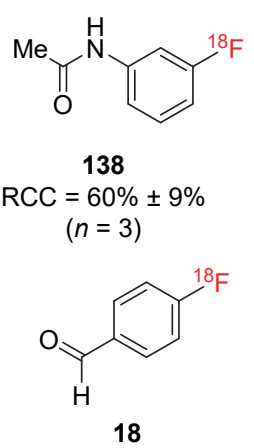

$\mathrm{RCC}=49 \% \pm 6 \%$ $(n=5)$

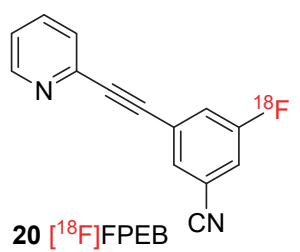

(20)

$\mathrm{RCC}=8 \% \pm 2 \%$

$(n=4)$
理论计算表明, 悉尼酮吸电子能力很强, 但是由于两环 上氢的存在阻碍了其自由旋转, 悉尼酮环无法与芳香环 共平面, 不能与芳烃完全共振, 限制了悉尼酮通过共振 离域稳定传入负电荷的能力, 这使得悉尼酮成为一个弱 阴离子稳定剂和优秀的离去基团, 减少了其他离去基团 离去产生的副产物发生. 底物芳香环上有吸电子取代基 时放射氟化过程以高放射化学转化率方式进行, 并且与 许多官能团兼容, 如硝基、氰基、酯、醚、砜、磺胺、 吡啶基、吗啉和酰胺, 以及难以标记的氟苯并呋喃类衍 生物. 特别是成功合成了 4 位悉尼酮取代的 ${ }^{18} \mathrm{~F}$ 氟苯类 化合物, 这一化合物可通过悉尼酮进一步发生环加成反 应，实现复杂生物分子的标记(Eq. 21).

\section{7 以有机金属化合物为前体进行氟-18 标记}

有机金属化合物反应活性、选择性好，对官能团耐 受性高, 这些特点使得有机金属化合物能成为一种优秀 的前体, 用于氟-18 放射性标记. 2011 年 Ritter 等 ${ }^{[45]}$ 报道 了一种以芳香钯(IV)复合物为前体亲核氟源参与的芳香 氟-18 标记方法. 两种钯复合物参与反应, 一种钯物种 与芳基硼酸或芳基硼酸酯类底物 ${ }^{[46]} 反$ 应生成了底物-钯 复合物 151, 另一钯物种则是预先捕获 ${ }^{18} \mathrm{~F}$ 氟离子形成 了 ${ }^{18} \mathrm{~F}$-钯复合物 $\mathbf{1 5 0}$, 两种钯物种反应后生成 $\operatorname{Pd}(\mathrm{IV})$ 芳 基氟化物配合物, 随后此物种再还原消除得到 ${ }^{18} \mathrm{~F}$ 标记 的目标产物(Scheme 7).

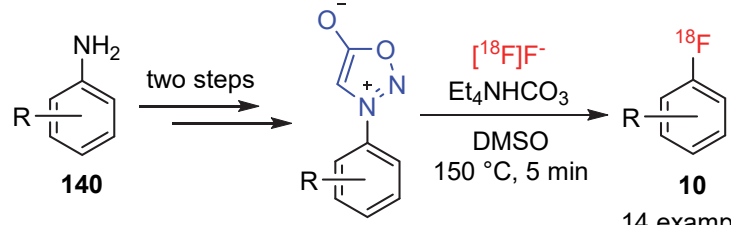

141

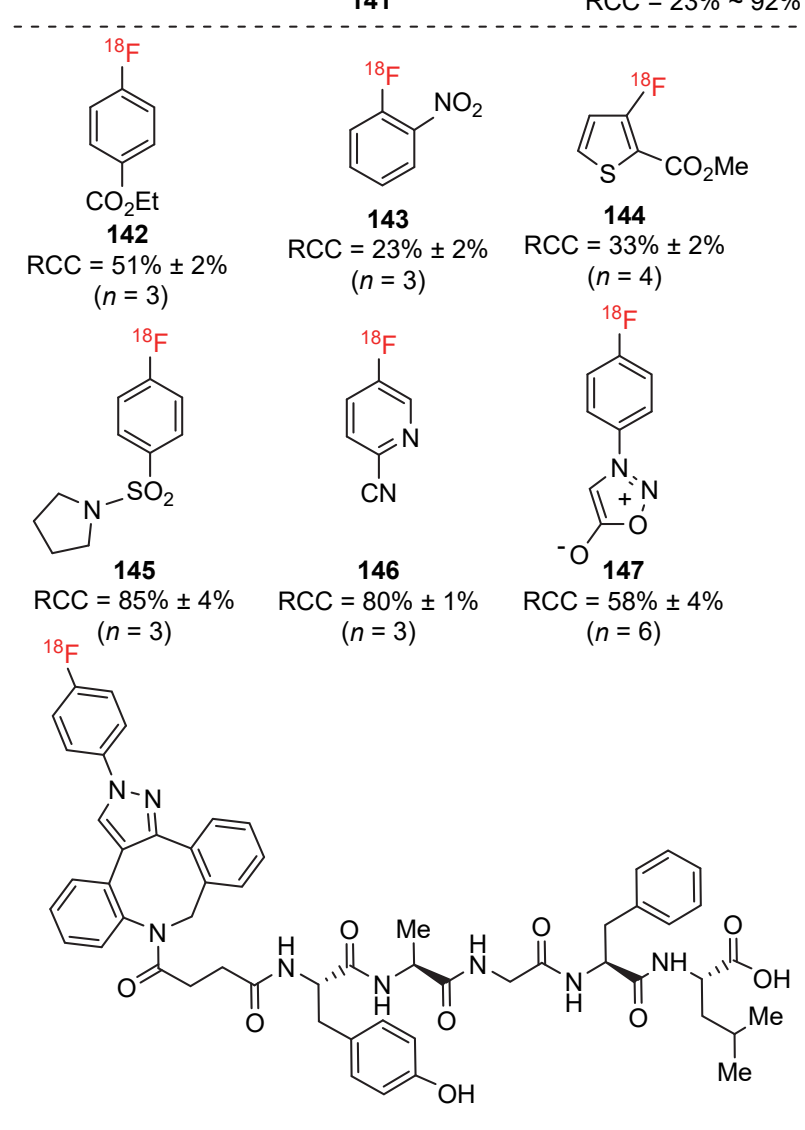

148 RCC > 97\%

详细的机理研究表明 [47], 由钯(IV)配合物介导的亲 电子氟化过程分为两步: (1) ${ }^{18} \mathrm{~F}$ 取代 4-甲基吡啶取代 $\mathrm{Pd}$ (IV)络合物中的 4-甲基吡啶生成 $\left.{ }^{18} \mathrm{~F}-\mathrm{Pd}(\mathrm{IV})\right)$ 复合物; (2) 亲核试剂(底物-Pd 复合物)与 ${ }^{18} \mathrm{~F}-\mathrm{Pd}(\mathrm{IV})$ 复合物的亲电反 应. 第一步中带相反电荷的 ${ }^{18} \mathrm{~F}$ 氟离子和钯配合物之间 形成了外球配合物, 因此具有很高的配位速率, 可以在 放射化学环境中的纳摩尔至微摩尔浓度下形成 ${ }^{18} \mathrm{~F}-\mathrm{Pd}$ (IV)复合物; 随后的氟-18 是通过 ${ }^{18} \mathrm{~F}-\mathrm{Pd}$ (IV)复合物的单 电子转移、 ${ }^{18} \mathrm{~F}$ 离子转移和第二次 $\mathrm{SET}$ 三个过程进行的. 这一机制研究对以后氟化物进行亲电 ${ }^{18} \mathrm{~F}$ 氟化提供了理 论支持和新的研究方向.

2012 年 Ritter 课题组 ${ }^{[48]}$ 报道了以芳香镍(II)复合物 为前体的放射性氟标记方法. 溴苯衍生物经两步反应得 到对水和硅胶稳定的底物-镍(II)复合物, 随后此前体在 氧化剂存在下，在室温使用含有 ${ }^{18} \mathrm{~F}$ 氟离子的水溶液在 一分钟即可完成放射性标记反应生成芳香氟-18 化合物, 通过该反应成功合成了在邻位、间位、对位存在富电子 


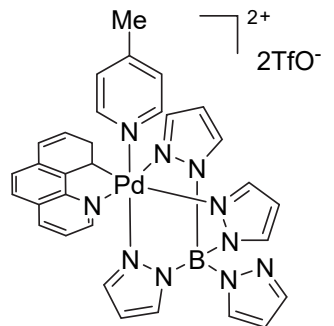

$$
\frac{\begin{array}{c}
{[18 \mathrm{~F}] \mathrm{F}^{-}} \\
\mathrm{K}_{2.2 .2 .}, \mathrm{K}_{2} \mathrm{CO}_{3}
\end{array}}{\text { Acetone, }}
$$

$23^{\circ} \mathrm{C}, 10 \mathrm{~min}$

149

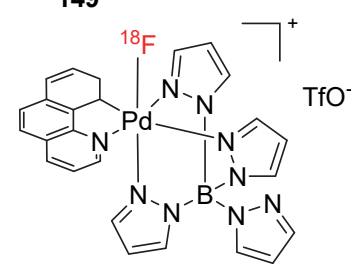

150
${ }^{18} \mathrm{~F}-[\mathrm{Pd}]$

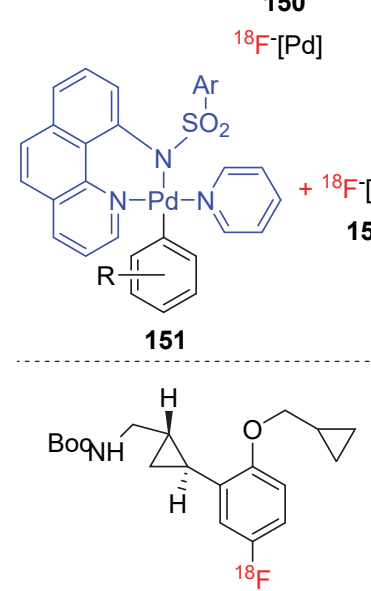

152

$\mathrm{RCY}=18 \% \pm 5 \%$

$(n=8)$

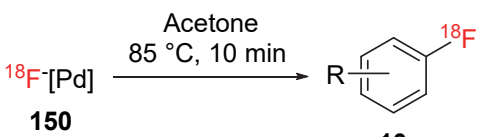

10

3 examples RCY $=10 \% \sim 33 \%$

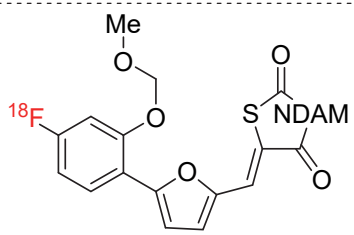

153

$\mathrm{RCY}=10 \% \pm 2 \%$

$(n=7)$<smiles>[Y16]C12CCC3c4ccc([15F])cc4CC[C@H]3[C@@H]1CCC2=O</smiles>

154

$\left[{ }^{18} \mathrm{~F}\right]$ Fluorodeoxyestrone $\mathrm{RCY}=33 \% \pm 7 \%(n=8)$

图式 7 以有机钯复合物为前体进行氟-18 标记

Scheme $7 \quad\left[{ }^{18} \mathrm{~F}\right]$ Fluorination of palladium aryl complexes

或吸电子取代的氟-18 取代苯及稠环芳香烃. 无需费时 的共沸干燥, 极大地简化了操作和缩短了反应时间, 这 一芳香镍(II)复合体为前体的放射性氟标记方法提高了 实用性，减少了反应时间(Eq. 22).

2014 年同一课题组 ${ }^{[49]}$ 改进了上述方法, 将干燥后 的 ${ }^{18} \mathrm{~F}$ 氟离子用碱 $\mathrm{TBAB}$ 洗脱至含缓冲酸对甲苯磺酸吡 啶鎓盐(PPTS)的混合体系中, 克服了前述方法在放射性 标记过程中镍复合物和氧化剂会发生竞争性水解的问 题，提高了放射化学收率.

2016 年, Murphy 等 ${ }^{[00]}$ 报道了芳基锡类的芳香亲核 氟-19 冷标记反应, 同年 Sanford 课题组 ${ }^{[51]}$ 将这一方法应
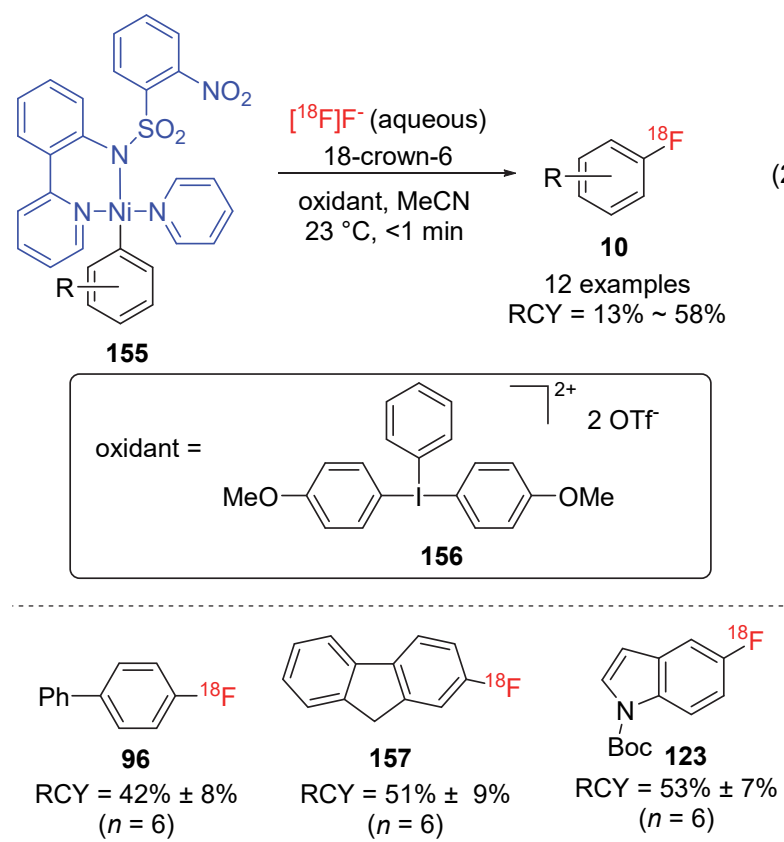<smiles>COC(=O)C(Cc1cc(OC(C)(C)C)c(OC(C)(C)C)cc1[PH3+])C(=O)OCc1ccccc1</smiles>

158

$\mathrm{RCY}=16 \% \pm 7 \%$ $(n=6)$

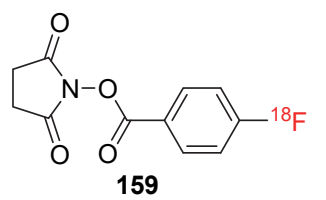

$\left[{ }^{18} \mathrm{~F}\right] \mathrm{SFB}$

$\mathrm{RCY}=21 \% \pm 4 \%$ $(n=6)$
用于热标记中. 反应在 $140{ }^{\circ} \mathrm{C}$ 下 2 equiv. $\mathrm{Cu}(\mathrm{OTf})_{2}, 15$ equiv. 吡啶及 $0.1 \mathrm{~mol} \cdot \mathrm{L}^{-1} \mathrm{DMA}$ 时得到最优反应条件. 锡烷上的烷基取代基对反应收率会产生重大影响. 总的 来说，三甲基取代的锡烷优于三正丁基取代的锡烷前 体, 这可能由于位阻较小时锡的转金属过程速度更快. 不同电性的底物均能得到较好的放射化学产率，适用于 一系列芳环、杂环和乙烯基锡烷前体，各类取代基均具 有较好的 RCCs; 邻位取代具有良好的耐受性, 邻位取 代的甲氧基和对位取代的标记结果相当(RCC 分别为 $57 \%$ 和 $48 \%$ ), 而其他多数底物亲核放射性氟化反应通 常在邻位取代芳香族底物时放射化学产率要低得多. 运 用这一方法以中等放射化学收率合成了多巴胺递质显 像剂 $\left[{ }^{18} \mathrm{~F}\right] \mathrm{F}-\mathrm{DOPA} 、 \mathrm{mGluR} 5$ 显像剂 $\left[{ }^{18} \mathrm{~F}\right] \mathrm{F}-\mathrm{PEB}$ 和 $5-\mathrm{HT}_{1 \mathrm{~A}}$ 受体显像剂 $\left[{ }^{18} \mathrm{~F}\right] \mathrm{MPPF}$ (Eq. 23).

\section{2 亲核氟源参与的芳环碳-氢键氟-18 化}

多年以来放射化学家们对氟-18 标记前体的开发表 明, 芳香环通过转变成前体而预功能化的方式是一种可 行且高效的方法. 但是, 对于某些复杂结构而言, 这一 方法增加了合成及分离的困难, 可能会制约其应用, 需 要有新的方法弥补这一缺陷. 研究者们尝试实现芳香烃 类底物碳一氢键的直接放射性氟化. 然而，由于 $\mathrm{C}\left(\mathrm{sp}^{2}\right)$ - 


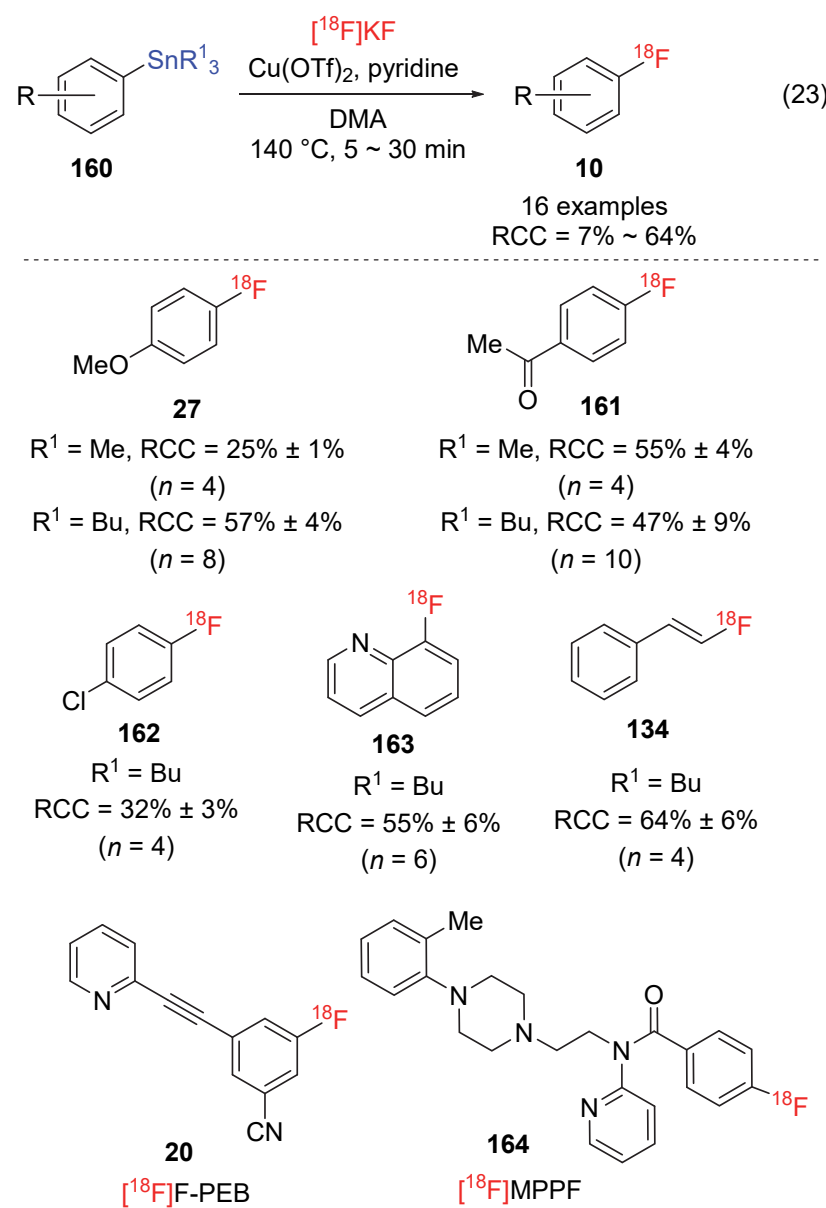

$\mathrm{R}^{1}=\mathrm{Bu}, \mathrm{RCC}=11 \% \pm 2 \% \quad \mathrm{R}^{1}=\mathrm{Me}, \mathrm{RCC}=33 \% \pm 4 \%$

$$
(n=4) \quad(n=4)
$$

$\mathrm{H}$ 键的惰性以及亲核型 ${ }^{18} \mathrm{~F}$ 氟源与大多数芳香烃底物之 间的电性相斥，该方法的仍然难以实现.

\section{1 导向基团参与的芳香碳氢键氟-18 化}

辅助基团及导向基团协助过渡金属催化反应引起 了放射化学家们的关注. 2013 年 Daugulis 课题组 ${ }^{[52]}$ 首次 报道了 $\mathrm{Cu}(\mathrm{II})$ 催化的亲核氟源存在下 8 -氨基喹啉导向基 团活化芳香环邻位 $\mathrm{C}\left(\mathrm{sp}^{2}\right)-\mathrm{H}$, 并与 $\mathrm{AgF}$ 发生亲核氟化 反应得到冷标记产物. 2019 年, Sanford 课题组 ${ }^{[53]}$ 改进了 这一方法, 并将其应用于芳香环氟-18 标记中. 该方法以 芳香酸-8-氨基喹啉酰胺为标记底物, $\mathrm{K}^{18} \mathrm{~F}$ 作为亲核氟 源, 在催化剂 $(\mathrm{MeCN})_{4} \mathrm{CuOTf}$ 和缚酸剂 1,8-二氮杂双环 (5.4.0)十一碳七烯(DBU)存在条件下, 在 $90{ }^{\circ} \mathrm{C} \sim 110{ }^{\circ} \mathrm{C}$ 反应 $30 \mathrm{~min}$ 即可得到中等的放射化学收率. 加入少量的 $N$-甲基吗啉(NMM)后可以进一步提高收率. 与底物以 酰胺形式结合的导向基团很容易裂解得到各类邻位氟18 标记的芳香羧酸类放射性药物, 也可以使用自动合 成模块以获取高比活放射性示踪剂. 芳环各位置上的吸 电子和电中性基团可以得到中等至较高的放化收率, 对 吡啶和吲哚等杂环类底物有效; 同时也对四种芳酸类药 物: 抗痛风药丙磺舒, $\mathrm{RAR}_{\beta 2}$ 激动剂 $\mathrm{AC} 261066$, RAR- alpha/beta 激动剂他米巴罗汀和蛋白表达修复药阿塔嫝 进行了氟-18 标记, RCCs 为 13\% 37\%(Eq. 24).
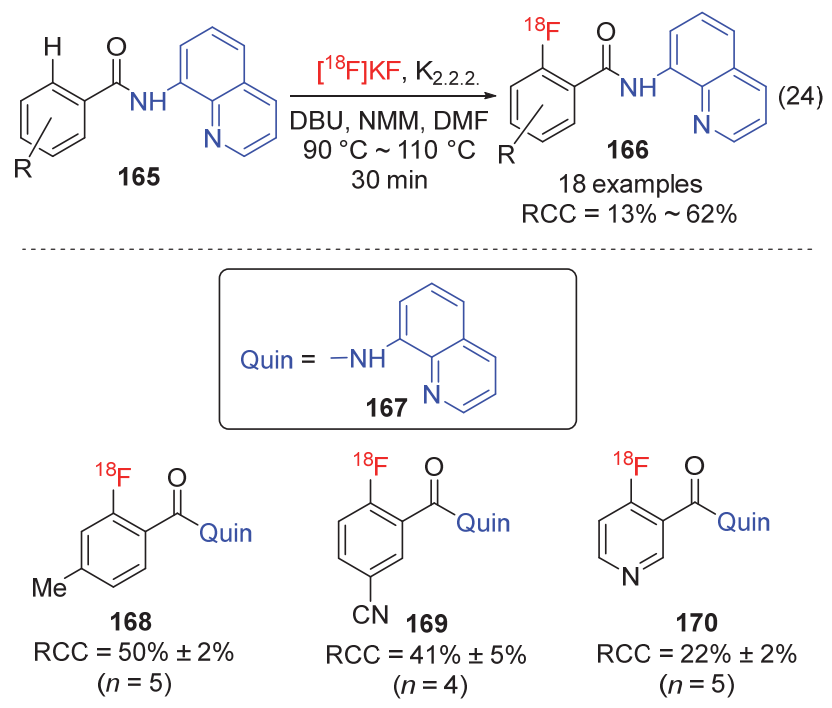<smiles>CCCN(CC)S(=O)(=O)c1ccc(C(=O)O)c(F)c1</smiles>

$\left[{ }^{18} \mathrm{~F}\right]$ Probenecid-Quin $\mathrm{RCC}=20 \% \pm 2 \%$ $(n=5)$

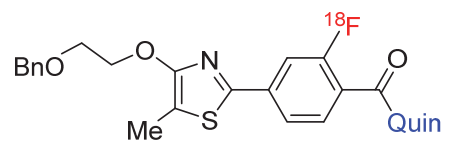

172

$\left[{ }^{18} \mathrm{~F}\right] \mathrm{AC} 261066-\mathrm{Quin}$ $\mathrm{RCC}=37 \% \pm 2 \%$ $(n=7)$

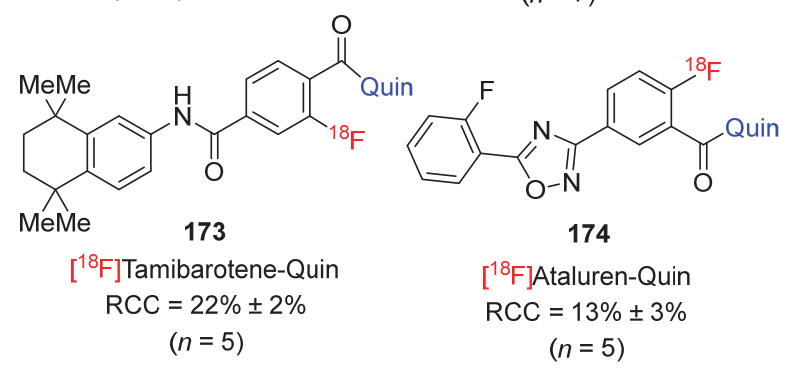

\section{2 光催化亲核氟源参与的碳-氢键氟-18 化}

2019 年, $\mathrm{Li}$ 课题组 ${ }^{[54]}$ 报道了一种温和的在蓝色光照 下芳环直接的氟- 18 标记反应. 在氧气气氛下, 低温 $\left(0{ }^{\circ} \mathrm{C}\right)$ 使用 $3.5 \mathrm{~W}$ 的 $450 \mathrm{~nm}$ 激光自上而下照射反应液 $30 \min$ (冷却至 $0{ }^{\circ} \mathrm{C}$, 以防止激光产生的热量损失溶剂), 即可得到较低至中等的放射化学收率. 一般来说芳环 氟-18 化发生在芳基阳离子自由基的最亲电位置上，而 对于 2 位和 4 位取代的芳烃，计算出的阳离子自由基亲 电性与观察到的放射化学收率之间几乎没有相关性, 因 此表明空间效应在确定位点选择性方面的贡献远大于 电性. 芳环上取代的吸电子基团、电中性基团、给电子 基团均能得到标记产物. 此方法也成功用于标记几种非 甾体抗炎药; 芬诺洛芬甲酯、氟比洛芬甲酯和 $O$-甲基水 杨酸甲酯(Eq. 25). 
机理研究表明反应过程是：叮啶类光催化剂(3,6,二叔丁基-9-均三甲苯基-10-苯基叫啶高氯酸盐)首先吸 收紫外光跃迁至激发态, 随后夺取芳环一个电子后变成 自由基, 再被氧气氧化为基态, 生成的氧自由基则被 2,2,6,6-四甲基哌啶氧化物(TEMPO)清除; 芳环被夺取 一个电子后生产的自由基阳离子被亲核氟源进攻生成 中间态自由基, 氧气夺取一个氢原子后即生成目标标记 产物, 过氧化物自由基则再被 TEMPO 清除(Scheme 8).

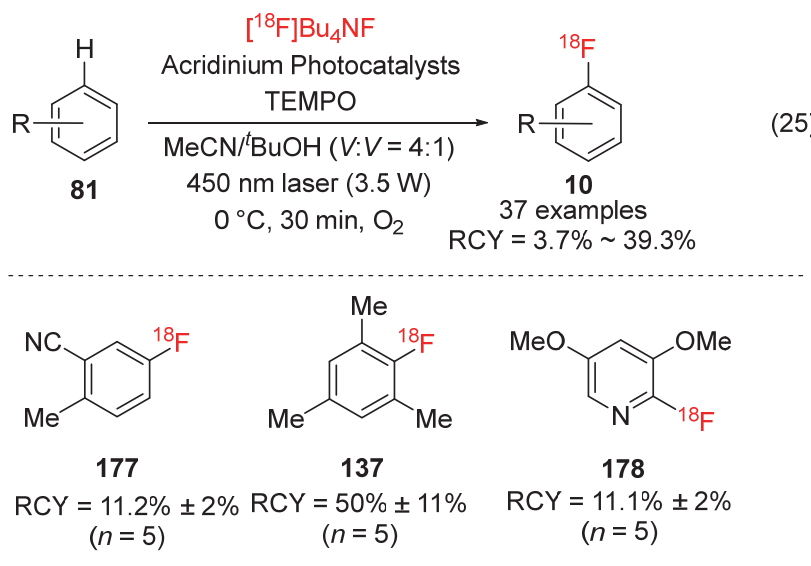<smiles>CC(=O)C(c1cccc(Oc2ccc(F)cc2)c1)[N+](=O)[O-]</smiles>

$\left[{ }^{18} \mathrm{~F}\right]$ Fenoprofen methyl ester $\mathrm{RCY}=39.6 \% \pm 1 \%$ $(n=5)$ $\left[{ }^{18} \mathrm{~F}\right]$ Flubiprofen methyl ester $\mathrm{RCY}=36.8 \% \pm 6 \%$ $(n=5)$

\section{4 总结与展望}

在过去的数十年间, 芳香化合物的氟-18 标记研究 发展迅速. 多种类型的标记前体不断被发现和优化, 碳 氢键活化和光催化等新型合成方法也在放射化学领域 得到了应用. 这些成果一方面是 PET 成像技术在药物研 究和临床应用的重要性日益突出, 越来越多的药学工作 者利用正电子标记技术研究常规药物的作用机制及代 谢历程; 另一方面是各类新型标记反应的发现及改进.

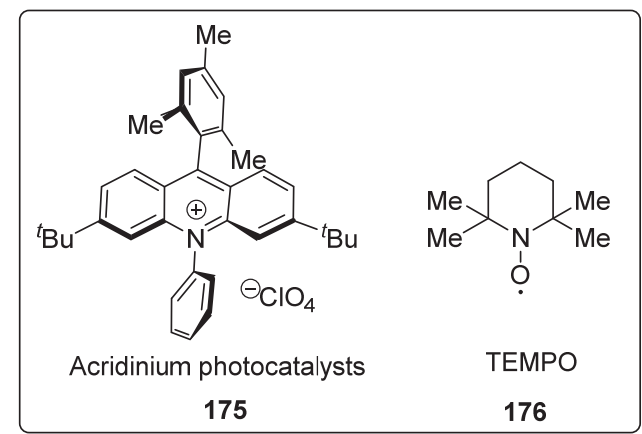<smiles>CCOC(=O)C(C)(C)Oc1ccc(Cl)cc1[N+](=O)[O-]</smiles>

181

$\left[{ }^{18} \mathrm{~F}\right]$ Clofibrate $\mathrm{RCY}=3.7 \% \pm 0.3 \%$ $(n=5)$<smiles>CCCC(=O)C(C)(OCC)Oc1ccc(C(=O)c2ccc(Cl)cc2)cc1Br</smiles>

182

$\left[{ }^{18} \mathrm{~F}\right]$ Fenofibrate $\mathrm{RCY}=5.6 \% \pm 0.4 \%$ $(n=5)$

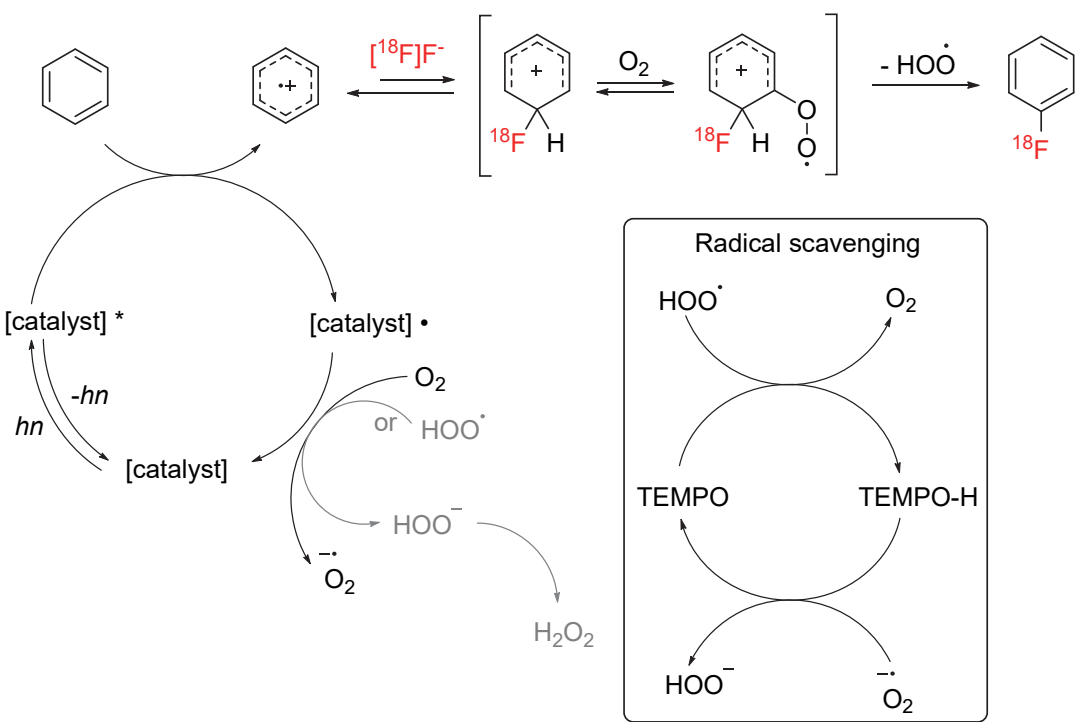

图式 8 光催化芳香环碳-氢键氟-18 化的机理研究

Scheme 8 Possible mechanism of $\left[{ }^{18} \mathrm{~F}\right]$ fluorination of arene $\mathrm{C}\left(\mathrm{sp}^{2}\right)-\mathrm{H}$ bonds via organic photoredox catalysis 
这些进步与发展突破了以往预留功能位点对于药物性 质的束缚, 为设计放射性药物提供了更广阔的空间. 随 着氟-18 标记技术在临床和临床前研究中越来越广泛的 应用，亲核氟源参与的芳香化合物标记将会得到持续发 展. 原有标记已不能满足需求, 迫切需要获得新型标记 方法.

长期以来, 放射性药物的发展一直受到放射性核素 及标记方法的严格限制, 发展新型标记方法显然更具前 景. 理想情况下, 好的标记反应具有广泛的官能团耐受 性, 并应与药物化学中最常见的构建单元兼容. 迄今为 止, 绝大多数的氟的热标记方法都是来源于氟-19 氟化 的相关研究. 将冷标记方法转换为热标记方法需要做进 一步优化, 以适应临床前和临床研究所需的放射性示踪 剂和放射性药物的合成要求. 在氟-18 标记研究中, 不 但要解决这类移植反应天然存在的前体合成纯化困难、 复杂前体普适性差等问题, 还需解决热标记特有的共沸 干燥、放射性物质的后处理以及自动化合成等环节造成 的收率下降问题. 尽管至今放射化学工作者已经进行了 大量的工作, 但氟热标记方法仍处于初步发展阶段.

展望未来的发展, 要解决诸多问题, 实现绿色、高 效、简便地合成放射性示踪剂和放射性药物, 首先发现 新型底物和碳氟键形成方法是实现反应普适性的关键; 其次利用自动合成程序控制热标记过程中繁琐而精细 的步骤, 实现标记方法的高重现性, 并贴近各类标记产 物实际的应用前景; 最后将底物拓展至各类复杂化合物 乃至生物大分子及生物制品, 是氟-18 标记反应的最终 目标.

\section{References}

[1] Piel, M.; Vernaleken, I.; Rosch, F. J. Med. Chem. 2014, 57(22), 9232.

[2] Matthews, P. M.; Rabiner, E. A.; Passchier, J.; Gunn, R. N. Br. J. Clin. Pharmacol. 2012, 73(2), 175.

[3] Xiong, K. L.; Yang, Q. W.; Gong, S. G.; Zhang, W. G. Nucl. Med. Commun. 2010, 31(1), 4.

[4] Dobrucki, L. W.; Sinusas, A. J. Nat. Rev. Cardiol 2010, 7(1), 38.

[5] Ametamey, S. M.; Honer, M.; Schubiger, P. A. Chem. Rev. 2008, 108(5), 1501.

[6] Fernandez, I.; Frenking, G.; Uggerud, E. J. Org. Chem. 2010, 75(9), 2971.

[7] Neumann, C. N.; Hooker, J. M.; Ritter, T. Nature 2016, 534(7607), 369.

[8] Beyzavi, M. H.; Mandal, D.; Strebl, M. G.; Neumann, C. N.; D'Amato, E. M.; Chen, J.; Hooker, J. M.; Ritter, T. ACS Cent. Sci. 2017, 3(9), 944

[9] Gao, Z.; Lim, Y. H.; Tredwell, M.; Li, L.; Verhoog, S.; Hopkinson, M.; Kaluza, W.; Collier, T. L.; Passchier, J.; Huiban, M.; Gouverneur, V. Angew. Chem. Int. Ed. 2012, 51(27), 6733.

[10] Mu, L.; Fischer, C. R.; Holland, J. P.; Becaud, J.; Schubiger, P. A.; Schibli, R.; Ametamey, S. M.; Graham, K.; Stellfeld, T.; Dinkelborg, L. M.; Lehmann, L. Eur. J. Org. Chem. 2012, 889.

[11] Sander, K.; Gendron, T.; Yiannaki, E.; Cybulska, K.; Kalber, T. L.; Lythgoe, M. F.; Arstad, E. Sci. Rep. 2015, 5, 9941.

[12] Chun, J. H.; Morse, C. L.; Chin, F. T.; Pike, V. W. Chem. Commun.
2013, 49(21), 2151.

[13] Pike, V. W.; Aigbirhio, F. I. J. Chem. Soc., Chem. Commun. 1995.

[14] Ross, T. L.; Ermert, J.; Hocke, C.; Coenen, H. H. J. Am. Chem. Soc. 2007, $129(25), 8018$.

[15] Chun, J. H.; Lu, S.; Lee, Y. S.; Pike, V. W. J. Org. Chem. 2010, $75(10), 3332$.

[16] Yuan, Z.; Cheng, R.; Chen, P.; Liu, G.; Liang, S. H. Angew Chem. Int. Ed. 2016, 55, 11882.

[17] Ichiishi, N.; Brooks, A. F.; Topczewski, J. J.; Rodnick, M. E.; Sanford, M. S.; Scott, P. J. Org. Lett. 2014, 16(12), 3224

[18] McCammant, M. S.; Thompson, S.; Brooks, A. F.; Krska, S. W.; Scott, P. J. H.; Sanford, M. S. Org. Lett. 2017, 19(14), 3939.

[19] Cardinale, J.; Ermert, J.; Humpert, S.; Coenen, H. H. RSC Adv. 2014, 4(33), 17293.

[20] Rotstein, B. H.; Stephenson, N. A.; Vasdev, N.; Liang, S. H. Nat. Commun. 2014, 5, 4365.

[21] Rotstein, B. H.; Wang, L.; Liu, R. Y.; Patteson, J.; Kwan, E. E.; Vasdev, N.; Liang, S. H. Chem. Sci. 2016, 7(7), 4407.

[22] Jakobsson, J. E.; Gronnevik, G.; Riss, P. J. Chem. Commun. 2017, 53(96), 12906.

[23] Haskali, M. B.; Telu, S.; Lee, Y. S.; Morse, C. L.; Lu, S.; Pike, V. W. J. Org. Chem. 2016, 81(1), 297.

[24] Ye, Y.; Schimler, S. D.; Hanley, P. S.; Sanford, M. S. J. Am. Chem. Soc. 2013, 135(44), 16292.

[25] Tredwell, M.; Preshlock, S. M.; Taylor, N. J.; Gruber, S.; Huiban, M.; Passchier, J.; Mercier, J.; Genicot, C.; Gouverneur, V. Angew Chem. Int. Ed. 2014, 53(30), 7751.

[26] Zhang, Z.; Zhang, C.; Lau, J.; Colpo, N.; Benard, F.; Lin, K. S. J. Labelled Comp. Radiopharm. 2016, 59(11), 467.

[27] Zhang, Z.; Lau, J.; Zhang, C.; Colpo, N.; Nocentini, A.; Supuran, C. T.; Benard, F.; Lin, K. S. J. Enzyme Inhib. Med. Chem. 2017, 32(1), 722.

[28] Blevins, D. W.; Kabalka, G. W.; Osborne, D. R.; Akula, M. R. Nat. Sci. 2018, 10(3), 125.

[29] Elie, J.; Vercouillie, J.; Arlicot, N.; Lemaire, L.; Bidault, R.; Bodard, S.; Hosselet, C.; Deloye, J. B.; Chalon, S.; Emond, P.; Guilloteau, D.; Buron, F.; Routier, S. J. Enzyme Inhib. Med. Chem. 2019, 34(1), 1 .

[30] Guibbal, F.; Meneyrol, V.; Ait-Arsa, I.; Diotel, N.; Patche, J.; Veeren, B.; Benard, S.; Gimie, F.; Yong-Sang, J.; Khantalin, I.; Veerapen, R.; Jestin, E.; Meilhac, O. ACS Med. Chem. Lett. 2019, 10(5), 743

[31] Mossine, A. V.; Tanzey, S. S.; Brooks, A. F.; Makaravage, K. J.; Ichiishi, N.; Miller, J. M.; Henderson, B. D.; Skaddan, M. B.; Sanford, M. S.; Scott, P. J. H. Org. Biomol. Chem. 2019, 17(38), 8701.

[32] Basuli, F.; Zhang, X.; Blackman, B.; White, M. E.; Jagoda, E. M.; Choyke, P. L.; Swenson, R. E. Molecules 2019, 24, 2389.

[33] Clemente, G. S.; Zarganes-Tzitzikas, T.; Dömling, A. H.; Elsinga, P. Molecules 2019, 24, 4210

[34] Lahdenpohja, S. O.; Rajala, N. A.; Rajander, J.; Kirjavainen, A. K. EJNMMI Radiopharm. Chem. 2019, 4, 28.

[35] Yuan, G.; Shoup, T. M.; Moon, S.-H.; Brownell, A.-L. RSC Adv. 2020, 10(42), 25223.

[36] Zhang, B.; Fraser, B. H.; Klenner, M. A.; Chen, Z.; Liang, S. H.; Massi, M.; Robinson, A. J.; Pascali, G. Chemistry 2019, 25(32), 7613.

[37] Preshlock, S.; Calderwood, S.; Verhoog, S.; Tredwell, M.; Huiban, M.; Hienzsch, A.; Gruber, S.; Wilson, T. C.; Taylor, N. J.; Cailly, T.; Schedler, M.; Collier, T. L.; Passchier, J.; Smits, R.; Mollitor, J.; Hoepping, A.; Mueller, M.; Genicot, C.; Mercier, J.; Gouverneur, V. Chem. Commun. 2016, 52(54), 8361.

[38] Taylor, N. J.; Emer, E.; Preshlock, S.; Schedler, M.; Tredwell, M.; Verhoog, S.; Mercier, J.; Genicot, C.; Gouverneur, V. J. Am. Chem. Soc. 2017, 139(24), 8267.

[39] Mossine, A. V.; Brooks, A. F.; Makaravage, K. J.; Miller, J. M.; Ichiishi, N.; Sanford, M. S.; Scott, P. J. Org. Lett. 2015, 17(23), 5780 .

[40] Zischler, J.; Kolks, N.; Modemann, D.; Neumaier, B.; Zlatopolskiy, 
B. D. Chemistry 2017, 23(14), 3251 .

[41] Zhang, X.; Basuli, F.; Swenson, R. E. J. Labelled Comp. Radiopharm. 2019, 62(3), 139.

[42] Wilson, T. C.; Xavier, M. A.; Knight, J.; Verhoog, S.; Torres, J. B.; Mosley, M.; Hopkins, S. L.; Wallington, S.; Allen, P. D.; Kersemans, V.; Hueting, R.; Smart, S.; Gouverneur, V.; Cornelissen, B. J. Nucl. Med. 2019, 60(4), 504.

[43] Guibbal, F.; Isenegger, P. G.; Wilson, T. C.; Pacelli, A.; Mahaut, D.; Sap, J. B. I.; Taylor, N. J.; Verhoog, S.; Preshlock, S.; Hueting, R.; Cornelissen, B.; Gouverneur, V. Nat. Protoc. 2020, 15(4), 1525.

[44] Narayanam, M. K.; Ma, G.; Champagne, P. A.; Houk, K. N.; Murphy, J. M. Angew. Chem. Int. Ed. 2017, 56(42), 13006.

[45] Lee, E.; Kamlet, A. S.; Powers, D. C.; Neumann, C. N.; Boursalian, G. B.; Furuya, T.; Choi, D. C.; Hooker, J. M.; Ritter, T. Science 2011, 334(6056), 639.

[46] Kamlet, A. S.; Neumann, C. N.; Lee, E.; Carlin, S. M.; Moseley, C. K.; Stephenson, N.; Hooker, J. M.; Ritter, T. PLoS One 2013, 8(3), e59187.

[47] Brandt, J. R.; Lee, E.; Boursalian, G. B.; Ritter, T. Chem. Sci. 2014, $5(1)$

[48] Lee, E.; Hooker, J. M.; Ritter, T. J. Am. Chem. Soc. 2012, 134(42), 17456

[49] Ren, H.; Wey, H. Y.; Strebl, M.; Neelamegam, R.; Ritter, T.; Hooker, J. M. ACS Chem. Neurosci. 2014, 5(7), 611.

[50] Gamache, R. F.; Waldmann, C.; Murphy, J. M. Org. Lett. 2016, $18(18), 4522$.

[51] Makaravage, K. J.; Brooks, A. F.; Mossine, A. V.; Sanford, M. S.; Scott, P. J. H. Org. Lett. 2016, $18(20), 5440$

[52] Truong, T.; Klimovica, K.; Daugulis, O. J. Am. Chem. Soc. 2013, 135(25), 9342.

[53] Lee, S. J.; Makaravage, K. J.; Brooks, A. F.; Scott, P. J. H.; Sanford, M. S. Angew. Chem. Int. Ed. 2019, 58(10), 3119.

[54] Chen, W.; Huang, Z.; Tay, N. E. S.; Giglio, B.; Wang, M.; Wang, H.; Wu, Z.; Nicewicz, D. A.; Li, Z. Science 2019, 364(6446), 1170.

(Fan, Y.) 